\title{
Leibniz, Kant und der moderne Symmetriebegriff
}

\author{
von Marco Giovanelli, Torino
}

\begin{abstract}
The paper analyses the significance of the modern concept of „symmetry“ for the understanding of the concept of „intuition“ in Kant's philosophy of geometry. A symmetry transformation or automorphism is a structure preserving mapping of the space into itself that leaves all relevant structure intact so that the result is always like the original, in all relevant respects. Hermann Weyl was the first to show that this idea can be drawn on Leibniz's definition of similarity: two figures are similar if they are only distinguishable through the simultaneous perception of them (comperceptio): , an automorphism carries a figure into one that in Leibniz's words is ,indiscernible from it if each of the two figures is considered by itself“ “. The author argues that, under the light of this definition, Leibniz's notion of „comperceptio“ turns out to play a similar role for the notion of „intuition“ in Kant's philosophy of space and time. Both cases are about the „difference between conceptual definition and intuitive exhibition", as Weyl puts it. This result has on the one hand an exegetical significance: it frees Kant's notion of „,intuition“ from every vague reference to the „visualisation“ of geometrical figures; on the other hand a theoretical one: it makes easy to compare Kant's philosophy of space and time with modern developments of sciences, in which as Weyl first showed, the concept of symmetry plays a fundamental role.
\end{abstract}

Keywords: Symmetry, Leibniz, Geometry, Incongruent Counterparts, Congruence, Similarity

\section{Einleitung: Hermann Weyls Definition des Symmetriebegriffs ${ }^{1}$}

Wenn man das Thema „Symmetrie“ in der Mathematik und in den Naturwissenschaften nochmals ${ }^{2}$ untersuchen will, erscheint es quasi unentbehrlich, auf das

1 Abkürzungen häufig zitierter Werke: C: Leibniz, Gottfried Wilhelm: Opuscules et fragments inédits de Leibniz. Extraits des manuscrits de la Bibliothèque royale de Hanovre par Louis Couturat. Hrsg. von Louis Couturat. Alcan 1903; LA: Leibniz, Gottfried Wilhelm: Sämtliche Schriften und Briefe. Hrsg. von der Deutschen Akademie der Wissenschaften zu Berlin; Akademie der Wissenschaften der DDR. Darmstadt 1923; LH: Leibniz, Gottfried Wilhelm: Die Leibniz-Handschriften der Königlichen Öffentlichen Bibliothek zu Hannover. Hrsg. von E. Bodemann. Hannover und Leipzig 1895; GM: Leibniz, Gottfried Wilhelm: Leibnizens mathematische Schriften. Hrsg. von Carl Immanuel Gerhardt. Halle 1850 GP: Leibniz, Gottfried Wilhelm: Die philosophischen Schriften von Gottfried Wilhelm Leibniz. Hrsg. von Carl Immanuel Gerhardt. Berlin 1875; PW: Leibniz, Gottfried Wilhelm: Philosophische Werke. Hrsg. von Artur Buchenau und Ernst Cassirer, 4 Bde. Leipzig 1996.

2 Unter den letzten Veröffentlichungen ist vor allem die Textsammlung zu erwähnen, die den status quaestionis am ausführlichsten darstellt: Katherine Brading und Elena Castellani: Symmetries in Physics: Philosophical Reflections. Cambridge, U.K./New York 2003. Eine weniger technische Darstellung findet man in Castellani, Elena: Simmetria e natura. Dalle armonie delle figure alle invarianze delle leggi. Roma/Bari 2002. Weitere wichtige Texte: La simmetria. Hrsg. von Evandro Agazzi. Bologna 1973; Genz, Henning: Symmetrie - Bauplan der Natur. München/Zürich 1987; Noël, Émile: La symétrie aujourd'hui. Paris 1989; Van Fraassen, Bas C.: Laws and Symmetry. Oxford/New York 1989; Henning 
klassische Buch Symmetry (1952) ${ }^{3}$ von Hermann Weyl, einem der größten Mathematiker des 20. Jahrhunderts, und auf die in ihm enthaltene Definition des Symmetriebegriffs Bezug zu nehmen. In der Antike, so Weyl, bedeutete „symmetrisch etwas wie wohlproportioniert“: ${ }^{4}$ Die Teile eines „symmetrischen“ Körpers, etwa einer Statue, sollten in einem bestimmten Verhältnis zueinander stehen, so dass das Gesicht ein Zehntel der Gesamtgröße ausmachen sollte, der Kopf ein Achtel, der Oberkörper ein Viertel usw. In der neueren Zeit heißt Symmetrie dagegen üblicherweise „bilaterale Symmetrie, die Symmetrie von links und rechts“. 5 Wenn man sagt, dass links und rechts „symmetrisch“ sind, ist gemeint, dass „die innere Struktur des Raumes uns, außer durch willkürliche Wahl, nicht gestattet, eine Links- von einer Rechtsschraube zu unterscheiden “. ${ }^{6}$ Ist die erste Definition willkürlich, da es vollkommen kulturabhängig ist, ob ein bestimmtes Verhältnis zwischen den Teilen und dem Ganzen wohlproportioniert aussieht oder nicht, zeigt dagegen Weyl, dass die zweite Auffassung einer präzisen mathematischen Behandlung unterworfen werden kann: „Diese bilaterale Symmetrie ist aber ein rein geometrischer und, im Gegensatz zu der oben besprochenen vagen Vorstellung von Symmetrie, ein absolut präziser Begriff“" ${ }^{7}$

Die bilaterale Symmetrie erscheint nämlich Weyl nur „als erster Fall eines geometrischen Symmetriebegriffs “. ${ }^{8}$ Ein Objekt im Raum heißt im Allgemeinen sym-

Genz und Roger Decker: Symmetrie und Symmetriebrechung in der Physik. Braunschweig 1991; Giora Hon und Bernard R. Goldstein: From summetria to Symmetry: The Making of a Revolutionary Scientific Concept. Dordrecht 2008. Unter den „Klassikern“ vgl. vor allem: Weyl, Hermann: Symmetry. Princeton, NJ 1952; Feynman, Richard Phillips: The Character of Physical Law. Cambridge 1965, Kap. IV; Wigner, Eugene Paul: Symmetries and Reflections: Scientific Essays of Eugene P. Wigner. Woodbridge 1979.

3 Wir zitieren aus der deutschen Ausgabe Weyl, Hermann: Symmetrie. Basel 1955.

4 A.a.O., 11.

5 Ebenda. Vgl. dazu Hon, Giora, From Proportion to Balance. The Background to Symmetry in Science. In: Studies in History and Philosophy of Modern Physics 36, 2005, Nr. 1, 21.

6 Weyl: Symmetrie, 24.

7 A.a.O., 12. Mathematisch werden Symmetrien als strukturinvariante Selbstabbildungen (Automorphismen) definiert. In der Geometrie ist eine Spiegelung ein Beispiel für einen Automorphismus, der die Form und die Größe einer Figur unveränderlich (invariant) lässt und nur die Orientierung ändert: „Was wir also mit der Behauptung meinen, daß links und rechts wesensgleich sind, ist die Tatsache, daß die Spiegelung an einer Ebene ein Automorphismus ist." (Weyl: Symmetrie, 26f.). Wie Weyl schreibt, ist ein Automorphimus „eine eineindeutige oder umkehrbar eindeutige Abbildung“ der Raumpunkte „auf sich selbst, die die Grundrelationen ungestört läßt" Weyl, Hermann: Philosophie der Mathematik und Naturwissenschaft. München 1990, 98. Die fundamentale Eigenschaft für Automorphismen ist, dass sie das bilden, was die Mathematiker eine „Gruppe“ nennen. Ein Beispiel für eine Transformationsgruppe sind die Bewegungen im Raum: das Resultat zweier aufeinanderfolgender Bewegungen lässt sich nämlich stets auch durch eine einzige Bewegung darstellen. In einer solchen Perspektive einer „Geometrie“ im abstrakten Sinne wird die Automorphismengruppe definiert. Sie untersucht alles, was gegenüber den Transformationen dieser gegebenen Gruppe invariant ist. Was hier besonders relevant ist, ist, dass, wie es zu zeigen ist, nach Weyl „Leibniz [...] erkannt [habe], daß dies die Idee ist, die dem geometrischen Begriff der Ähnlichkeit zugrunde liegt“ (Weyl: Symmetrie, 26).

8 Weyl: Symmetrie, 13. 
metrisch, wenn man es nicht nur spiegeln, sondern auch z.B. drehen, verschieben, vergrößern oder verkleinern kann, so dass man am Ende der Operation nicht sagen kann, ob die Figur, die man vor sich hat, das Urbild oder das Nachbild ist. Urbild und Nachbild können erst unterschieden werden, wenn man sie miteinander vergleicht oder im Allgemeinen mit etwas, das nicht verschoben, gespiegelt oder gedreht wurde, d.h. in Bezug auf etwas Asymmetrisches. ${ }^{9}$ Richard Feynman hat diese Auffassung der Symmetrie vielleicht am besten zusammengefasst: „Laut der ausgezeichneten Definition Professor Weyls, eines Mathematikers, ist ein Ding symmetrisch, wenn es eine Möglichkeit gibt, es zu verändern, und es hinterher doch wieder so aussieht wie vorher". ${ }^{10}$

Von dieser klassischen Definition von Symmetrie ausgehend will man versuchen nachzuweisen, dass, wenn man die Beispiele in Betracht zieht, die Leibniz und Kant verwenden, um die Natur der Geometrie zu veranschaulichen, es nicht gewagt zu sein scheint zu behaupten, dass sie alle von demselben Grundproblem ausgehen, das heutzutage mit dem Wort „Symmetrie“ bezeichnet wird. Leibniz und Kant versuchen zu zeigen, dass der Unterschied zwischen einer Figur und ihrem symmetrischen Abbild (z. B. der Unterschied zwischen einer rechtsorientierten und linksorientierten Schraube, zwischen einer Figur und ihrem verkleinerten Modell usw.), durch keine begriffliche Beschreibung der Merkmale, die eine Figur besitzt und die andere nicht, sondern erst durch einen anschaulichen Vergleich auftreten kann. Eine Figur und ihr symmetrisches Abbild können nach Leibniz' Ausdrucksweise erst durch „Komperzeption“ (comperceptio) unterschieden werden, oder in Kants Terminologie, wenn sie unmittelbar in der „Anschauung“ (intuitio) verglichen werden. Obwohl die „Leibnizianer“ immer wieder der Polemik Kants ausgesetzt waren, scheint es von diesem Standpunkt aber möglich zu behaupten, dass der Anschauungsbegriff in Kants Raumtheorie eine ähnliche Rolle wie der Komperzeptionsbegriff in jener von Leibniz spielt. Besser gesagt wird er verwendet, um das gleiche Problem des Unterschiedes von „symmetrischen “ Figuren zu lösen, das zuerst Leibniz gestellt hat.

Das hat meines Erachtens erstens eine rein exegetische Bedeutung: Die Rolle der „Anschauung“ in Kants Philosophie der Geometrie wird damit von vielen ihrer Zweideutigkeiten befreit: Die „Anschauung“ in Kants Raumlehre bedeutet nicht die Evidenz der geometrischen (Euklidischen) Axiome und auch nicht die bildliche Darstellbarkeit der geometrischen Figuren, wie oft gemeint wurde. ${ }^{11}$ Die Gegen-

9 Vgl. Rosen, Joseph: Symmetry Rules: How Science and Nature Are Founded on Symmetry. Berlin/Heidelberg 2008, $49 \mathrm{ff} ., 144 \mathrm{ff}$.

10 Wir zitieren aus der deutschen Ausgaben: Feynman, Richard Phillips: Vom Wesen physikalischer Gesetze. München 1993, 106.

11 Diese sind zwei übliche, z.B. von der Seite der Neupositivisten, polemische Motive gegen Kants Raumlehre. Es wird einerseits behauptet, dass die geometrischen Axiome a priori in der „reinen Anschauung“ gegeben sind: Vgl. z.B. Rudolph Carnap: „Diese einfache Tatsachen der Geometrie, so sagte Kant, sehen wir unmittelbar. Wir begreifen ihre Wahrheit intuitiv“. In: Carnap, Rudolph: Einführung in die Philosophie der Naturwissenschaft. München 1974, 128. Andererseits, z.B. von Hans Reichenbach, wird oft gesagt, dass Kant für Notwendigkeit von „schlecht gezeichneten Figuren“ in geometrischen Beweisen plädiert habe (vgl. Reichenbach, Hans: Die Philosophie der Raum-Zeit-Lehre. In: Ders., 
überstellung von „Begriff“ und „Anschauung“ bedeutet vielmehr, dass die Geometrie die Einführung einer Form von anschaulicher Verschiedenheit zwischen geometrischen Figuren voraussetzt, nämlich zwischen einer Figur und ihrem symmetrischen Abbild, das auf die Begriffsbildung der traditionellen Logik nicht zurückzuführen ist. Die Möglichkeit, Kants Raumlehre vom Standpunkt des modernen Symmetriebegriffes zu betrachten, hat aber zweitens auch eine theoretische Tragweite: Kants Wissenschaftstheorie kann nämlich damit auf einfachste Weise mit den heutigen Entwicklungen der Naturwissenschaften verglichen werden, in denen verschiedene Formen von „Symmetrien“, wie zuerst gerade Weyl gezeigt hat, unbezweifelbar eine grundlegende Rolle spielen. ${ }^{12}$

Selbstverständlich kann keiner dieser beiden Aspekte hier ausführlich behandelt werden. Die Bedeutung der vorliegenden Arbeit steht vielmehr an der Schnittstelle dieser beiden Grundrichtungen, aus der meines Erachtens einige klassische Interpretationsprobleme von Kants Raumlehre in einem anderen Licht erscheinen können.

\section{Leibniz und die Vorgeschichte des Symmetriebegriffs}

\subsection{Leibniz’ Definition der Ähnlichkeit}

Die Darstellung Weyls ist nicht nur klassisch; für die vorliegende Arbeit macht sie sich vor allem dafür verdient, dass sie den modernen Symmetriebegriff historisch und systematisch auf Leibniz' Definition von Ähnlichkeit zurückgeführt hat: „Leibniz“, so Weyl, „[hat] dem geometrischen Begriff der Ähnlichkeit die folgende philosophische Wendung gegeben [...]: Ähnlich, sagt er, sind zwei Dinge, die ununterscheidbar sind, wenn jedes für sich betrachtet wird “. ${ }^{13}$ Dieser Hinweis Weyls kann in den Texten Leibniz' selbst ausführlich bestätigt werden. ${ }^{14}$

Gesammelte Werke in 9 Bänden. Hrsg. von Andreas Kamlah und Maria Reichenbach, Braunschweig/Wiesbaden 1977, Bd. 2, 11; in diesem Sinne würde „Anschauung“" etwas wie „Visualisation" bedeuten, wie das Wort in der englischen Übersetzung von den Werken von Reichenbach wiedergegeben wird (vgl. Reichenbach, Hans: The Philosophy of Space and Time. Hrsg. von Maria Reichenbach. New York 1958, 83, Anm. 2). Jakko Hintikka war vielleicht der erste, der betonte, dass „Anschauung“ mit „Anschaulichkeit“ in keiner Weise zu tun hat: „There is nothing, intuitive“ about intuitions so defined. Intuitivity means simply individuality “ (Hintikka, Jaakko: Kant on mathematical Method. In: Kant's Philosophy of Mathematics. Hrsg. von Carl J. Posy. Dordrecht/Boston/London 1992, 26-27).

12 Für eine jüngste Stellungnahme vgl. z.B. Weinberg, Steven: Conceptual Foundations of the Unified Theory of Weak and Electromagnetic Interactions. In: Origin of Symmetries. Hrsg. von Colin D. Froggatt und Holger B. Nielsen. Singapore [u. a.] 1991, 215-223 oder Gross, David J.: The Role of Symmetry in Fundamental Physics. In: Proceedings of the National Academy of Sciences 93, 1996, 14256-14259.

13 Weyl: Symmetrie, 23.

14 Man hat hier selbstverständlich nicht den Anspruch, das geometrische Raumproblem bei Leibniz ausführlich zu behandeln. Eine jüngste und umfassende Gesamtdarstellung findet man in De Risi, Vincenzo: Geometry and Monadology: Leibniz's analysis situs and Philosophy of Space. Basel/Boston 2007. Grundlegend für die folgende Darstellung bleibt aber immer noch Schneider, Martin: Funktion und Grundlegung der Mathesis Universalis. 
Jede geometrische Figur hat nach Leibniz eine Qualität (oder Form, z.B. die Form eines Viereckes) und eine Quantität (oder Größe: es gibt größere oder kleinere Vierecke). Figuren können also nach „Qualitatem et Quantitatem, sive Magnitudinem et Formam “ [der Qualität und der Quantität, der Größe und der Form] (LA VI.4a, 514) charakterisiert werden. Zwei Figuren, die dieselbe Quantität haben, heißen „gleich“ (z. B. ein Dreieck und ein Viereck mit demselben Flächeninhalt); wenn sie aber dieselbe Qualität haben, sind sie „ähnlich“ (z.B. zwei Vierecke von verschiedener Größe; vgl. z.B. GM 5: 179; De analysi situs; PW 1: 50).

Die Unterscheidung zwischen Qualität und Quantität wird von Leibniz folgendermaßen erklärt: Zur Erfassung der Quantität müssen die Gegenstände, die man vergleicht, unmittelbar nebeneinander gegeben sein oder doch durch irgendeine Art der Vermittlung, d.h. durch Beziehung auf ein drittes Objekt als Maßstab, tatsächlich einander gegenübergestellt werden können. Die Qualität hingegen stellt dem Betrachter etwas dar, was sich in einem Gegenstand, auch wenn man ihn allein betrachtet, für sich erkennen und weiterhin zum Vergleich zweier Gegenstände brauchen lässt, ohne dass es nötig ist, die Vergleichsobjekte unmittelbar oder mittelbar miteinander in Beziehung zu setzen.

Das gleichseitige Dreieck ist zum Beispiel von einem ungleichseitigen einfach zu unterscheiden, auch wenn beide nicht zusammen betrachtet werden. Aber wenn man das größere von zwei gleichseitigen Dreiecken bestimmen will, ist der Vergleich mit dem anderen nötig. Man kann nämlich kein Attribut, keine Eigenschaft in einem finden, die im anderen nicht gefunden werden kann. Wenn man sie aber zusammen betrachtet, wird stets der Unterschied deutlich, dass eines nämlich größer ist (vgl. GM 5: 155):

Similia sunt, in quibus per se singulatim consideratis inveniri non potest quo discernantur, ut duo sphaerae vel circuli (vel duo cubi aut duo quadrata perfecta) A et B. Ut si solus oculus sine aliis membris fingantur, nunc esse intra sphaeram A nunc intra sphaeram B, non poterit eas discernere, sed poterit si ambas simul spectet, vel si secum membra alia corporis aliamve mensuram introrsum afferat, quam nunc uni nunc alteri applicet. Itaque ad similia discernenda opus est vel compraesentia eorum inter se, vel tertii cum singulis successive. [At in dissimilibus aliqua partium proportio notata in uno, quae non notatur in altero, sufficit ad discernendum sigillatim]. (GM 7: 30; Initia mathematica, De quantitate) [Ähnlich sind diejenigen, in denen, wenn sie einzeln betrachtet werden, nichts gefunden werden kann, das sie voneinander unterscheidet, wie zwei vollkommene Kugeln oder Kreise (oder zwei Würfel oder zwei vollkommene Quadrate) A und B. Wenn man sich vorstellt, mit dem bloßen Auge, ohne andere Körperteile, bald in der Kugel A, bald in der Kugel B zu sein, kann man sie nicht

In: Studia Leibnitiana Sonderheft 15 (Leibniz Questions de Logique), 1988, 162-182. $\mathrm{Zu}$ erwähnen sind auch Freudenthal, Hans: Leibniz und die Analysis situs. In: Studia Leibnitiana 4/2, 1972), 61-69; Münzenmayer, Hans Peter: Der Calculus Situs und die Grundlagen der Geometrie bei Leibniz. In: Studia Leibnitiana 11, 1979, 274-300; Mainzer, Klaus: Symmetrien der Natur. Ein Handbuch zur Natur- und Wissenschaftsphilosophie. Berlin [u.a.] 1988, 142ff; Wallwitz, Georg Graf: Strukturelle Probleme in Leibniz Analysis Situs. In: Studia Leibnitiana 23, 1991, 111-118; Mugnai, Massimo: Leibniz' Theory of Relations. Stuttgart 1992, 89 ff. Im Weiteren sind auch folgende Klassiker nützlich: Couturat, Louis: La logique de Leibniz d'après des documents inédits. Hildesheim 1961, Kap. IX und Cassirer, Ernst: Leibniz' System in seinen wissenschaftlichen Grundlagen. In: Ders., Gesammelte Werke. Hrsg. von Marcel Simon, Hamburg 1998, Bd. 1, Kap. III. 
unterscheiden; man kann es aber, wenn man sie zusammen beobachtet oder wenn man andere Körperteile in die Kugel bringt oder ein anderes Maß, das an dem einen sowie an dem anderem angelegt wird. Dagegen ist in unähnlichen Figuren eine gewisse Proportion genug, die in einem gefunden werden kann und im anderen nicht, um sie einzeln zu unterscheiden.]

Infolgedessen sagt Leibniz üblicherweise, dass diejenigen Figuren „ähnlich“ sind, die, ohne zusammen betrachtet zu werden (compercipere), nicht unterschieden werden können, d.h. nur entweder unmittelbar, durch Kongruenz oder aktuelle Koinzidenz eines Teils einer Figur mit dem der anderen, oder mittelbar, d.h. durch einen Maßstab, der an beiden Figuren oder an deren Teilen angewandt werden kann. Dies genügt, um festzustellen, ob etwas kongruent ist oder nicht. Dagegen braucht man zwei unähnliche Figuren nur zusammen zu denken (concogitare), sie aber nicht in einem Vergleichsakt zugleich anzuschauen.

Die Berufung Leibniz’ auf die „Komperzeption“, auf ein anschauliches Vergleichen ${ }^{15}$ beider Figuren, erscheint zunächst als die Einführung eines rein empirischen oder sogar psychologischen Fremdelements in die Geometrie. Leibniz meint aber die prinzipielle Unmöglichkeit, den Unterschied zwischen den ähnlichen Figuren A und B festzustellen, wenn man keinen direkten Vergleich durchführen kann. Wenn man eine einzelne Figur betrachtet, ist es nicht möglich zu sagen, ob es um A oder B geht.

Wenn man sich beispielsweise vorstellt, dass Gott alle Erscheinungen in uns und um uns herum in einem geschlossenen Raum verkleinert, die Proportionen aber aufrecht erhält, so würde alles genauso wie zuvor aussehen, und wir könnten den vorherigen Status vom späteren nicht unterscheiden, wenn wir nicht aus der Sphäre der verhältnismäßig verkleinerten Dinge, d.h. aus jenem Raum, wieder herauskommen; die Verkleinerung wäre nämlich erst im Vergleich mit den nicht verkleinerten Sachen bemerkbar (vgl. GM 7: 277). Wenn also Gott alles verhältnismäßig verkleinern würde, wäre es prinzipiell unmöglich, eine Unterscheidung vorzunehmen, beziehungsweise hätte es gar keinen Sinn, von einer solchen Unterscheidung zu reden, denn wenn alle Sachen der Welt, die wir beobachten, in derselben Proportion verkleinert würden, ist es klar, dass man die Änderung nicht bemerken könnte (vgl. auch GM 1: 180; Brief an Galloys, 1677):

At si quemadmodum alibi jam dixi Deus omnia mutaret proportione eadem servata perisset nobis omnis mensura, nec possemus scire quantum res mutatae sint, quoniam mensura nulla certa definitione comprehendi adeoque nec memoria retineri potest, sed opus est reali ejus conservatione. Ex quibus omnibus discrimen inter magnitudinem et speciem inter quantitatem et qualitatem elucere arbitror. (GM 7: 276; Specimen Geometriae Luciferae) [Wenn dagegen auf irgendeine Weise nun Gott alles ändern würde, indem er die Proportionen aufrechterhält, verlieren wir jedes Maß und können nicht wissen, um wie viel sich die Sachen verändert haben, denn es ist unmöglich, eine sichere Definition des Maßes zu bestimmen oder sie im Gedächtnis zu behalten, sondern es ist notwendig, sie wirklich aufrechtzuerhalten. Aus diesem allem glaube ich den Unterschied zwischen Größe und Art, zwischen Quantität und Qualität zu erhellen.]

15 „[C]omparaison intuitive“ (Couturat: La logique de Leibniz d'après des documents inédits, 412). 
Was man beobachtet, ist nicht die Veränderung der Länge an sich, sondern nur die Veränderung des Verhältnisses zu einer anderen Länge, die als Maßstab gewählt wurde. Wenn sich also alles in denselben Proportionen verändern würde, hätte man kein Mittel, diese Veränderung festzustellen. Beide Zustände wären derselbe Zustand, und der Unterschied könnte erst bemerkt werden, wenn wir den Bezug auf etwas hätten, das sich nicht vergrößert oder „verkleinert“ hat (vgl. GM 5: 154; 7: 276).

So ist es unmöglich zu erkennen, was der „Fuß“ oder die „Elle“ ist, wenn man nicht ein wirklich gegebenes Objekt als Maßstab zugrunde legt und es sodann nacheinander mit verschiedenen Gegenständen zusammenbringt. Was ein Fuß ist, kann daher durch keine Definition vollständig erklärt werden, denn man kann nicht das Gemeinsame aller ein Fuß langen Strecken finden; sagt man, der Fuß besteht aus zwölf Zoll, so erhebt sich dieselbe Frage für die Maßeinheit „Zoll“ usw. Man kann daher nur sagen, dass eine bestimmte Strecke „die gleiche Länge“ wie eine direkt ausgewiesene Strecke hat, die man willkürlich als „Standardfuß“ gewählt hat. Hier besteht also ein Unterschied zwischen dem „Geben“ eines individuellen Objekts durch eine anschauliche Ausweisung und der begrifflichen Beschreibung, die seine Merkmale definiert: „Enim alius possit esse circulus pedalis, alius semipedalis etc., tamen pedis nulla dari potest definitio, sed opus est typo aliquo fixo et permanente“ (GM 7: 276). [In der Tat, obwohl es einen einfüßigen Kreis, einen halbfüßigen, usw. geben kann; kann man keine Definition von Fuß geben, sondern braucht einen festen und dauerhaften Standard].

\subsection{Leibniz' Definition des Kongruenzbegriffs}

Wie eben erläutert wurde, kann man ohne Komperzeption also zwei ähnliche geometrische Figuren nicht unterscheiden. Wenn wir aber zwei Figuren betrachten, die nicht nur ähnlich, sondern auch gleich sind, d.h. kongruent, ${ }^{16}$ ist es nicht genug, die beiden Figuren zusammen anzuschauen, um sie zu unterscheiden, denn in dem Falle wären sie koinzident:

Si vero duae res non tantum sunt similes sed et aequales, id est si sint congruae etiam simul perceptas non discernere possum, nisi loco id est, nisi adhuc aliud assumant extra ipsas et observem ipsas diversum habere situm ad tertium assumtum. Denique si ambo simul in eodem sint loco, jam nihil habere me amplius quo discriminentur (GM 5: 155; Charachteristica geometrica). [Wenn jedoch zwei Dinge nicht nur ähnlich, sondern auch gleich sind, d.h. dass sie kongruent sind, auch wenn man sie zusammen anschaut, können sie nicht unterschieden werden, außer durch die Lage, d.h. insoweit man etwas anderes außerhalb von ihnen annimmt und man beobachtet, dass sie eine unterschiedliche Lage in Bezug auf dieses Dritte einnehmen. Denn wenn sie in demselben Ort sind, bleibt mir nichts übrig, das sie unterscheidet.]

16 Zu Leibniz’ Kongruenzbegriff vgl. A.a.O., 310; Münzenmayer: Der Calculus Situs und die Grundlagen der Geometrie bei Leibniz. 296ff; Mugnai: Leibniz' Theory of Relations, 89; Cassirer: Leibniz' System in seinen wissenschaftlichen Grundlagen. 140ff; De Risi: Geometry and Monadology: Leibniz's analysis situs and Philosophy of Space, $132 \mathrm{ff}$. 
Bei zwei ähnlichen Figuren mit derselben Qualität, die sich an demselben Ort befinden, verhält es sich also so: Eine koinzidiert nur mit einem Teil der anderen und kann damit von dieser unterschieden werden. Wenn sie auch die gleiche Quantität hätten, müssten sie an verschiedenen Stellen sein, um verschieden zu erscheinen, sonst wären sie nicht nur zum Teil sondern völlig koinzident. Man muss also die Figuren auf ein drittes Objekt legen, in Bezug auf welches sie verschiedene Lagen einnehmen:

Congrua sunt quae sola comperceptione cum tertio discerni possunt [...] Congrua itaque sunt, quorum qualitas et quantitas eadem est, et quae tamen positione discernuntur. (LA 6.4a: 565) [Kongruent sind diejenigen, die nur durch die Komperzeption mit einem Dritten unterschieden werden können. [...] Kongruent sind diejenigen, deren Quantität und Qualität dieselbe ist und die nur durch die Lage unterschieden werden können.]

Kongruent sind also Figuren, die, wenn sie verschieden sind, nur in Bezug auf etwas Äußeres unterschieden werden können wie zwei Quadrate etwa C und D, denn sie sind zur selben Zeit an verschiedenen Orten oder Lagen. Wenn man aber nur ein Quadrat isoliert betrachtet, kann man nicht mehr sagen, ob es um C oder $\mathrm{D}$ geht, da alles, was für C allein gilt, auch für D gilt und umgekehrt. Die Verschiedenheit besteht also nur in der gegenseitigen Stellung zu einem bestimmten Bezugsobjekt, das man direkt aufgewiesen hat. Leibniz sagt daher, dass kongruente Figuren nur noch solo numero, nur weil sie zwei sind, bzw. referentia ad externa unterschieden werden können (vgl. GM 7:275f.).

Auch in diesem Fall hat die Komperzeption mit einem dritten Objekt nicht nur eine psychologische Bedeutung; es geht um die prinzipielle Unmöglichkeit, $\mathrm{C}$ und D zu unterscheiden, wenn man sie isoliert betrachtet. Leibniz benutzt deswegen nochmals dasselbe Gedankenexperiment: Wenn man das ganze Universum an einen anderen Ort verschieben würde, könnte man nicht feststellen, ob diese Änderung stattgefunden hat, da es prinzipiell kein Objekt außerhalb des Universums gibt, in Bezug auf welches die Bewegung festgestellt werden könnte:

De dire que Dieu fasse avancer tout l'univers, en ligne droite ou autre, sans y rien changer autrement, c'est encor une supposition chimerique. Car deux etats indiscernables sont le même etat, et par consequent c'est un changement qui ne change rien (GP 7: 373; Streitschriften zwischen Leibniz u. Clarke. Leibniz' viertes Schreiben). [Die Annahme, dass Gott das Universum in gerader oder in anderer Richtung verrücken lasse, ohne darin sonst die geringste Änderung vorzunehmen, ist wiederum chimärisch. Denn zwei unterscheidbare Zustände sind ein und derselbe Zustand, diese wäre also eine Änderung, die nichts ändert.] (PW 1: 104f.)

Wenn man diese berühmte Stelle im Kontext der Leibniz'schen Reflexionen über die Geometrie liest, lässt sich feststellen, dass es hier nicht nur um die Relativität des Raumes geht, sondern vielmehr um die subtilere Behauptung, dass der Raum verschiebungssymmetrisch ist. Das tritt noch deutlicher auf, wenn man bedenkt, dass dasselbe gilt, wenn man das Universum in sein Spiegelbild transformiert. Wenn man alles von „rechts“ nach „links“ verkehren und ansonsten alles unverändert lassen würde, könnte man keinen Unterschied zwischen beiden Universen feststellen. Denn auch hier gilt; ohne Zuhilfenahme eines dritten Körpers lässt sich nicht zwischen den Eigenschaften eines Körpers und jenen des Spiegelbildes unterscheiden. So Leibniz in einer gleichwohl berühmten Stelle: 
[I]l est impossible qu'il y ait une raison, pourquoy Dieu, gardant les mêmes situations des corps entre eux, ait placé les corps dans l'espace ainsi et non pas autrement, et pourquoy tout n'a pas eté mis à rebours (par exemple) par un échange de l'orient et de l'occident [...] ces deux etats, l'un tel qu'il est, l'autre supposé à rebours, ne differeroient point entre eux. (GP 7:364; Streitschriften zwischen Leibniz u. Clarke. Leibniz' drittes Schreiben). [Es ist] unmöglich, einen Grund dafür anzugeben, weshalb Gott die Körper, die Beibehaltung ihrer Abstände und gegenseitigen Lagebeziehungen vorausgesetzt, gerade an diese bestimmte Raumstelle und nicht an eine andere gesetzt hat; warum nicht alles durch einen Umtausch von Westen nach Osten umgekehrt angeordnet worden ist [...] so sind eben diese beiden Zustände, der ursprüngliche und seine Umkehrung, in nichts voneinander verschieden.] (PW 1: 94)17

Gerade wie bei der Definition von „Fuß“ oder „Elle“ ist es daher unmöglich, im geometrischen Raum „rechts“ und „links“ zu unterscheiden, ohne auf ein direkt aufgewiesenes asymmetrisches Objekt (z. B. den menschlichen Körper) Bezug zu nehmen, das z.B. als rechtsorientiert dekretiert wurde. Leibniz scheint das zu behaupten, z. B. in dieser Textpassage: „Sed dextrum a sinistro discerni non potest [...] nisi facto ipso, seu perceptione, dum ab uno latere motum commodiorem quam ab alio homines experiuntur" [Aber es ist unmöglich rechts von links zu unterscheiden [...], wenn nicht für die Tatsache selbst oder die Wahrnehmung, indem die Menschen erfahren, dass an einer Seite die Bewegung bequemer als auf der anderen ist] (C S. 478; Phil VII, D, II, 2, f. 30).

\subsection{Identität und Äquivalenz}

Wenn man behauptet, dass das Universum und sein Spiegelbild oder sein vergrößertes Modell usw. „dasselbe “ Universum sind, bedeutet „dasselbe“ nicht eine absolute Identität, sondern eine bloße Gleichwertigkeit. In der Geometrie sucht man nämlich nicht nach „une identité, une chose qui soit véritablement la même“ [nach einer Identität, einem Ding, das wahrhaft dasselbe wäre] (GP 7: 401; Streitschriften zwischen Leibniz u. Clarke. Leibniz' fünftes Schreiben; PW 1: 135), sondern nach einer bloßen aequivalentia oder aequipollentia. ${ }^{18}$

17 Nicht alle Interpreten sind sich darüber einig, dass es in Leibniz' Beispiel um eine Spiegelung geht. Es könnte sich nämlich auch um eine Drehung um $180^{\circ}$ handeln (vgl. z.B. Mates, Benson: The Philosophy of Leibniz Metaphysics and Language. New York, NY 1986, 233). Die These der Spiegelung wird dagegen von Weyl (Weyl: Symmetrie, 28f.) und Max Jammer (Jammer, Max: Concepts of Space the History of Theories of Space in Physics. Cambridge, Massachusetts 1954, 223-226) vertreten. John Earman hält beide Interpretationen für korrekt (vgl. Earman, John: World Enough and Space-Time: Absolute vs. Relational Theories of Space and Time. Cambridge, Mass. 1989, 173). Wenn man an den Fall von Buridans Esel denkt (s.u. S. 10), scheint es aber plausibel, dass Leibniz hier eine Spiegelung meinte. Vgl. dazu auch Khamara, Edward J.: Space, Time, and Theology in the Leibniz-Newton Controversy. Frankfurt 2006, $83 \mathrm{f}$.

18 Vgl. dazu die klassische Interpretation von Gottlob Frege: Frege, Gottlob: Die Grundlagen der Arithmetik eine logisch-mathematische Untersuchung über den Begriff der Zabl. Hildesheim 1977, 78 f.; vgl. Angelelli, Ignacio: On Identity and Interchangeability in Leibnitz and Frege. In: Notre Dame Journal of Formal Logic 8/1-2, 1967, 94-100: 96f. und Ishiguro, Hidé: Leibniz's Philosophy of Logic and Language. Ithaca, N.Y. 1972, 17-34. Im Allgemeinen über dieses Thema vgl. auch Weyl: Philosophie der Mathematik und Natur- 
Die Sätze, die die Euklidische Geometrie von einem bestimmten Gebilde entwickelt, bleiben z.B. unverändert, wenn wir die absolute Lage dieses Gebildes im Raum wechseln, wenn wir die absoluten Größen im selben Verhältnis wachsen oder abnehmen lassen. Eine Figur kann also „statt der anderen“ benutzt oder kann von der anderen ersetzt werden, ohne die Gültigkeit der Sätze der Geometrie in Gefahr zu bringen. In der Euklidischen Geometrie bilden z. B. Kreise, die sich nur durch ihre Durchmesserlänge unterscheiden, nicht eine verschiedene, sondern dieselbe Figur, denn die absolute Größe der Linien betrifft keine Sätze der euklidischen Geometrie (vgl. GM 7: 276; Specimen geometriae luciferae). Die absolute Größe gehört also zu den sinnlichen Merkmalen, da sie nur durch die Kompräsenz (vgl. GM 1: 180) des willkürlich fixierten materiellen Maßstabs bestimmbar ist: „Similia, quae sibi substitui possunt salva qualitate seu ita discerni nequeant, nisi simul spectentur" (GM 7: 196) [Ähnlich sind die, die sich bei Wahrung der Qualität überall ersetzen können oder sie können nicht unterschieden werden, wenn man sie nicht zusammen beobachtet].

Dieser Indifferenz der Euklidischen Gebilde gegen alle absoluten Größenunterschiede entspricht jener der absoluten Lage. Die Sätze, die die Geometrie von einem bestimmten Gebilde entwickelt, bleiben unverändert, wenn dieses Gebilde seine absolute Lage im Raum wechselt. So sind auch zwei Dreiecke, die eine bloße Lageverschiedenheit aufweisen, „dasselbe“ Dreieck. Sie können nämlich bei Wahrung der Quantität und der Qualität ersetzt werden, d.h. sie können genau denselben Raum einnehmen oder zur Deckung gebracht werden, ohne irgendeine andere Änderung als die Stellenversetzung (vgl. GM 2: 20-25) zu vollziehen: "Congrua sunt quae sibi substitui possunt in eodem loco“ (GM 5: 172) [Kongruent sind die, die sich in demselben Ort ersetzen können]. Die Idee der „Ersetzbarkeit“ oder Deckungsmöglichkeit, die Idee, dass eine Figur an die Stelle einer anderen gebracht werden kann, bedeutet also, dass es die Geometrie mit Eigenschaften der Figuren zu tun hat, die von ihrer Position im Raum unabhängig sind. ${ }^{19}$

Der Raum ist also für Leibniz „sibi congruus“ und auch „sibi similis“ (LH 34.1: 14, Bl. 23 retro $)^{20}$. Leibniz betont nämlich, dass die Teile des Raumes nicht nur zueinander kongruent (wie die Teile einer Kugeloberfläche), sondern auch zueinander ähnlich (wie die Teile einer Ebene) sind. Die Kongruenz aller Teile des Raumes ist also die geringste Bedingung für seine Uniformität. Wenn sie alle nicht kongruent wären, könnten sie sich noch weniger ähnlich sein, d.h. kongruente Teile darstellen.

In den Beziehungen von Kongruenz tritt also für Leibniz der Gedanke der „Einheit des Raumes“ auf, der selbst innerlich überall gleichförmig ist. Die vollkom-

wissenschaft, 25. Im Allgemeinen über Symmetrie und Äquivalenz vgl.: Castellani, Elena: Symmetry and equivalence. In: Symmetries in Physics: Philosophical Reflections. Hrsg. von Katherine Brading und Elena Castellani. Cambridge, U.K./New York. 2003, 422-433.

19 Vgl. Frege: Die Grundlagen der Arithmetik eine logisch-mathematische Untersuchung über den Begriff der Zahl, 76 ff.; Für eine jüngere Darstellung vgl. in: Ishiguro: Leibniz's Philosophy of Logic and Language, 17-34.

20 Der Text ist von De Risi: Geometry and Monadology: Leibniz's analysis situs and Philosophy of Space, 582-585 herausgegeben. Hier: 583. 
mene Gleichförmigkeit des Raumes wird nämlich damit ausgedrückt, dass alle Punkte miteinander kongruent, d.h. gleich und ähnlich sind („omnia puncta congruere inter se [...] adeoque et similia atque si loqui licet, aequalia esse “) oder, was für Leibniz dieselbe Bedeutung hat, in demselben Raum sind („omnia puncta sunt in eodem spatio“ (GM 5: 144; vgl. auch (GM 2: 23; Leibniz an Huygens 8. September 1879; PW 1: 58 f.). Es gibt also nur einen indifferenzierten Raum, und alle möglichen Figuren sind nur mögliche Einteilungen desselben Raumes (vgl. GP 7: 21; PW 1: 40).

\subsection{Leibniz und die universelle Verknüpfung aller Dinge}

Eine solche „Einheit des Raumes“, d.h. die Tatsache, dass alle Teile des Raumes gleich und ähnlich sind, oder dass die Möglichkeit einer bloßen Lageverschiedenheit von Figuren besteht, ist für Leibniz alles andere als selbstverständlich. Dies ist erklärbar, wenn man sich das vor Augen führt, was eine der wichtigsten Voraussetzungen der leibnizschen Philosophie ist, d.h. das, was man das „Prinzip der Fülle“ des Universums genannt hat oder die Idee des universellen Zusammenhangs aller Dinge. ${ }^{21}$ In der Natur existiere nichts derart „Getrenntes“, das nicht eine Beziehung zu etwas anderem einschlösse. Die Änderung eines Dinges setzt also nach Leibniz die Änderung aller anderen voraus (vgl. GP 6: 617; Monadologie; PW 2: 614).

Leibniz vergleicht deshalb das Universum mit einem Fluidum oder mit einem Ozean (vgl. auch GP 5: 179f.; 7: 567), in dem sich jede Bewegung verbreitet so wie sich eine Welle nach und nach allen Teilen mitteilt, obwohl immer weniger bemerkbar ist; jede geringe Änderung in einem Körper erstreckt ihre Wirkung auf die benachbarten Körper und demzufolge von einem zum anderen ins Unendliche. Auf dieselbe Weise kann jeder Körper durch die Veränderung aller anderen eine Beeinflussung erleiden; da er unendlich teilbar ist, kann er durch die verschiedenen Bewegungen ihrer unendlich vielen Teile eine Spur der Bewegung aller anderen Dinge in sich enthalten.

Wenn man auf die These der universellen Verknüpfung aller Dinge verweist, muss man also sofort bemerken, dass die Kongruenz für Leibniz nur im „Reich des Möglichen“ vorkommen kann. Im real existierenden Universum kann man nicht zwei nur durch die Position verschiedene Figuren finden; es gibt immer irgendeine Bestimmung, die eine individuelle Figur besitzt und die andere nicht:

Aussi la notion de la sphere en general est incomplete ou abstraite, c'est à dire on n'y considere que l'essence de la sphere en general ou en theorie sans avoir égard aux circonstances singulieres $[\ldots]$ mais la notion de la sphere qu'Archimede a fait mettre sur son tombeau est accomplie [...]. [O]utre la forme de la sphere, il y entre la matiere dont elle est faite, le lieu,

21 Vgl. Mugnai, Massimo: Der Begriff der Harmonie als metaphysische Grundlage der Logik und Kombinatorik bei Johannes Heinrich Bisterfeld und Leibniz. In: Studia Leibnitiana 5, 1973, 43-73: 49f; Rutherford, Donald: Leibniz and the Rational Order of Nature. Cambridge/New York, NY 1995, 36-40; Plaisted, Dennis: Leibniz on Purely Extrinsic Denominations. Rochester, NY 2002, 47-68. 
le temps, et les autres circonstances, qui par un enchainement continuel envelopperoient enfin toute la suite de l'univers, si on pouvoit poursuivre tout ce que ces notions enferment. (GP 2: 39; Remarques sur la lettre de M. Arnaud) [Auch der Begriff der Kugel im Allgemeinen ist unvollständig oder abstrakt, d.h. man betrachtet damit nur die Essenz der Kugel im Allgemeinen oder theoretisch [...], ohne die besonderen Umstände daran zu beachten [...]. Aber der Begriff der Kugel, die Archimedes auf sein Grab setzen ließ, ist vollständig [...]. $[\mathrm{N}]$ eben die Form der Kugel [tritt] die Materie, aus der sie gemacht ist, der Ort, die Zeit und die anderen Umstände, die durch einen beständigen Zusammenhang schließlich die ganze Folge des Universums enthalten würden, wenn man all das weiterverfolgen könnte, was diese Begriffe enthalten. $]^{22}$

In der Geometrie kann sich also eine Figur, ohne ihre Quantität und Qualität zu ändern, statt an diesem auch an jedem beliebigen anderen Ort im Raum befinden, in dem sie gleich und ähnlich bleibt, d.h. ohne irgendeine andere Veränderung, außer jener des Ortes, vorzunehmen. In der konkreten Realität ist keine „reine“ Ortsveränderung möglich (vgl. C Phil 14, c, 7; 9 f.). Jede Ortsbewegung muss nämlich immer eine Veränderung im Ding, das sich bewegt, hervorbringen, obwohl wir sie wegen der Verworrenheit unserer Sinne nicht bemerken können (vgl. GP 2: 43).

Die Folge dieser Betrachtungen ist, wie man ahnen kann, der „Satz der Identität des Ununterscheidbaren“ (Principium identitatis indiscernibilium). Der Satz besagt nämlich, dass die in der Geometrie geltenden Kongruenzbeziehungen in der Wirklichkeit versagen. In der abstrakten Welt der Geometrie gilt: „Congrua itaque sunt, quorum qualitas et quantitas eadem est, et quae tamen positione discernuntur.“ (LA 6.4a, 565) [Kongruent sind diejenigen, deren Qualität und Quantität gleich ist und die bloß durch die Lage unterschieden werden können]; in der konkreten Realität aber sind zwei nur durch die Lage zu unterscheidende Figuren unmöglich: „Hinc fieri nequit in natura ut duo corpora sint perfecte simul aequalia et similia“, „quae loco differunt.“ (GP 2: 250; Leibniz an de Volder) [In der Natur kann es demnach nicht vorkommen, daß zwei Körper einander vollkommen gleich und ähnlich sind [und nur] der Lage nach von einander verschieden.] (PW 2: 504). ${ }^{23}$ In der Wirklichkeit trifft man nämlich immer auf einzelne Dinge; die Kugel auf dem Grab des Archimedes ist eine ganz bestimmte, gerade „diese“. In der Geometrie ist dagegen jede Kugel immer nur ein Exemplar, das mit jeder anderen kongruenten Kugel völlig gleichwertig ist. So darf die Identität konkreter Individuen nicht mit der Kongruenz abstrakter Figuren verwechselt werden. Von einem abstrakten Standpunkt aus mag es also eine bloße numerische bzw. Lagenverschiedenheit geben; konkret ist aber die Verschiedenheit der Dinge immer mehr als numerisch (vgl. GP 5: 51; vgl. auch C Phil 14, c, 7, S. 8 ff.).

22 Leibniz, Gottfried Wilhelm und Antoine Arnauld: Philosophischer Briefwechsel: Der Briefwechsel mit Antoine Arnauld, französisch-deutsch. Hrsg. von Reinhard Finster. Hamburg 1997, 83-85.

23 Das Thema soll hier natürlich nicht abschließend behandelt werden. Mehr dazu in: Chernoff, Fred: Leibniz's Principle of the Identity of Indiscernibles. In: The Philosophical Quarterly 31/123, 1981, 126-138: 134f; Mates: The Philosophy of Leibniz Metaphysics and Language, 133; Mugnai: Leibniz' Theory of Relations, 89; Piro, Francesco: Vicissitudes de deux oeufs. Principe de raison et principe des indiscernables dans les premiers écrits de Leibniz. In: La monadologie de Leibniz. Genèse et contexte. Hrsg. von Enrico Pasini. Milano 2005, 3-29. 
In der abstrakten Geometrie kann eine Figur zerschnitten werden, „en sorte que tout soit egal et semblable de part et d'autre; comme une Ellipse et toute figure dans le plan, du nombre de celles que j'appelle amphidextres, peut être mipartie ainsi, par quelque ligne droite que ce soit qui passe par son centre" (GP 6: 130; Essais de Theodicée, Premiere partie) [derart, dass auf beiden Seiten alles gleich und ähnlich ist wie eine Ellipse und jede ebene Figur, die zu den von mir als ,amphidextra' bezeichneten Figuren gehört, durch eine gerade, ihren Mittelpunkt schneidende Linie derart halbiert werden kann] (PW 3: 122); dagegen sind die Teile des wirklichen Universums „infiniment variées; ainsi jamais le cas arrivera, où tout sera parfaitement egal et frappera egalement de part et d'autre“ (GP 3: 403; Leibniz an Coste. 19 Decembr. 1707) [unendlich vielfältig; es würde also nie ein Fall eintreten, in dem alles völlig gleich wäre und würde auf der einen und anderen Seite sich gleich zeigen.]. Behauptet man also, dass links und rechts in einer von Leibniz geprägten Ausdrucksweise ununterscheidbar sind, so soll damit ausgedrückt werden, dass sie für die innere Struktur des Raumes vollkommen gleichberechtigt sind und dass außer durch willkürliche Wahl, einen Akt anschaulicher Aufweisung, es nicht gestattet ist, begrifflich links und rechts zu unterscheiden. In der konkreten Realität aber sind sie nie vollkommen gleichwertig. Der Esel Buridans kann sich immer zwischen zwei Heuhaufen entscheiden, weil er sich nie im Fall einer vollkommenen Indifferenz befindet (vgl. GP 3: 402; 6: 571; 3: 168; 5: 106; 6: 329; 6: 563f.). ${ }^{24}$

Ein völlig einheitlicher Raum, wo alle Teile gleich und ähnlich sind, drückt aber nichts anderes aus als eine ideale „Möglichkeit“. Der davon unterschiedene Charakter des „Wirklichen“ liegt dagegen in seiner unendlichen Differenzierung. ${ }^{25}$ Es gibt nicht zwei Teile der Materie, die wahrhaft „dieselben“ sind. Die tiefere Zergliederung fördert vielmehr überall innere Unterschiede zutage, kraft derer jeder gegebene räumliche Inhalt als ein schlechthin einmaliger und einziger erscheint. Der abstrakte Raum ist also zwar „quelque chose d'uniforme absolument“ [etwas durchaus Gleichförmiges], in dem „un point de l'espace ne differe absolument en rien d'un autre point de l'espace“ (GP 7: 364; Streitschriften zwischen Leibniz und Clarke. Leibniz' drittes Schreiben) [jeder seiner Punkte von einem beliebigen anderen Punkt in nichts verschieden [ist]] (PW 1: 94). In der konkreten Realität aber „il y a partout des varietés actuelles et jamais une parfaite uniformité, ny deux pieces de matiere entierement semblables l'une à l'autre“ (GP 7:563) [gibt es überall eine aktuelle Vielfalt und niemals eine vollkommene Gleichförmigkeit und zwei Materiestücke sind niemals vollkommen ähnlich] ${ }^{26}$ :

Les parties du temps ou du lieu, prises en elles mêmes, sont des choses ideales; ainsi elles se ressemblent parfaitement, comme deux unités abstraites. Mais il n'en est pas de même de deux Uns concrets, ou de deux temps effectifs, ou de deux espaces remplis, c'est à dire,

24 Vgl. dazu Savage, Reginald Osburn: Real alternatives, Leibniz's metaphysics of choice. Dordrecht/Boston 1998, 11, 83, 84, 85, 94, 99, 178.

25 Vgl. Mugnai, Massimo: Astrazione e realtà: saggio su Leibniz. Milano 1976, $140 \mathrm{f}$.

26 Vgl. auch „spatium absolutum uniforme es seu ubique sibili simile (congruum) (LH 35.1: 12 Bl. 6 verso; in: De Risi [Hrsg.]: Geometry and Monadology: Leibniz's analysis situs and Philosophy of Space, 608. (Vgl. auch a.a.O. 179 f.). 
veritablement actuels (GP 7: 395; Streitschriften zwischen Leibniz u. Clarke; Leibniz' fünftes Schreiben). [Die Teile der Zeit und des Ortes sind an und für sich etwas Ideelles, gleichen sich daher vollkommen, wie zwei abstrakte Einheiten. Anders verhält es sich dagegen mit zwei konkreten Einheiten, zwei tatsächlichen Zeiten, oder zwei erfüllten, d.h. wahrhaft wirklichen Räumen.] (PW 1: 127f.)

In der von uns verwendeten Ausdrucksweise könnte man sagen, dass der leere Raum einen sehr hohen Grad von Symmetrie hat: Jeder Punkt ist jedem anderen gleich, und es gibt keinen Wesensunterschied zwischen den verschiedenen, von einem Punkt ausgehenden Richtungen, d.h. jeder Punkt und jede Richtung kann statt des beziehungsweise der anderen benutzt werden. Der wirkliche Raum ist dagegen höchst asymmetrisch, nicht nur in dem Sinne, ${ }^{27}$ dass er, um den Ausdruck Leibniz' zu verwenden, nicht „amphidexter“ ist, sondern allgemein, weil er unendlich variiert und in allerkleinsten Aspekten und Facetten differenziert ist, ${ }^{28}$ obwohl wir wegen der „Verworrenheit" unserer Sinne diese Unterscheidungen zumeist nicht bemerken können (vgl. C, Math 1: 5, c). „L'uniformité entière dans une partie du temps, du lieu ou de la matière“ [die völlige Einförmigkeit in einem Zeit-, Orts- oder Stoffteile] sind also bloß „fictions des philosophes qui viennent de leur notions incompletes “ [Phantasiegebilde der Philosophen, die aus ihren unvollständigen Begriffen stammen], weil „la matière (c'est à dire le melange des effects de l'infini environnant) fasse toujours quelque exception “ [die Materie, d.h. die Mischung der Wirkungen des uns umgebenden Unendlichen, immer eine gewisse Ausnahme macht] (GP 5: 50; PW 4: 14). Gleichförmig ist für Leibniz nur das Mögliche, nicht das Wirkliche.

\section{Der Symmetriebegriff bei Kant}

Was bisher gezeigt wurde, lässt sich so zusammenfassen: für Leibniz gibt es in der abstrakten Geometrie die Möglichkeit, zwei Gegenstände als unterschieden (z.B. weil sie verschiedene Orte einnehmen) zu erkennen, obgleich die Gegenstände in ihren Eigenschaften (Qualität und Quantität) vollkommen übereinstimmen (es gibt in einem nichts, das es im anderen nicht gibt). ${ }^{29}$ Wenn zwei Gegenstände dagegen in der konkreten Wirklichkeit verschieden sind, gibt es immer etwas, welches dem einen zukommt und dem anderen nicht. Die Kongruenz geometrischer Figuren, die miteinander vollkommen äquivalent sind, kann also nicht mit der Identität von konkret individuellen Dingen gleichgesetzt werden.

27 Vgl. dazu Mahnke, Dietrich: Leibnizens Synthese von Universalmathematik und Individualmethaphysik. Halle a.d.S., 16

28 Vgl. dazu: Mainzer, Klaus: G. W. Leibniz: Principles of Symmetry and Conservation Law. In: Symmetries in Physics (1600-1980). Proceedings of the 1st International Meeting on the History of Scientific Ideas held at Sant Feliu de Guixols, Catalonia, Spain, September 20-26, 1983. Hrsg. von Manuel G. Doncel. Barcelona 1987, 69-75: 25; Breger, Herbert: Symmetry in Leibnizean Physics. In: The Leibniz Renaissance: International Workshop (Firenze, 2-5 giugno 1986). Hrsg. von Paolo Rossi. Firenze 1989, 23-43.

29 Vgl. Reidemeister, Kurt: Raum und Zahl. Berlin 1957, 65 f. 
Die bisher durchgeführte Darstellung von Leibniz' Raumlehre konnte selbstverständlich solche Aspekte nicht in allen Details berücksichtigen. Sie wollte vielmehr auf der einen Seite zeigen, wie Leibniz das Grundproblem der „Symmetrie“ eingeführt hat, indem er die Möglichkeit eines bloß „anschaulichen“ Unterschiedes zwischen geometrischen Figuren zugelassen hat. Auf der anderen Seite wurden die Prämissen gesetzt, um zu erklären, wie dieses Grundproblem erst in einer verzerrten Form von Kant erfasst werden konnte, d.h. in der Form die Leibniz' „Nachfolger [...] in ihre Leibniz-Wolffianische Lehrgebäude ein[trugen] “ (KrV, B 329).

Die Leibnizianer des 18. Jahrhunderts scheinen nämlich diese Gegenüberstellung zwischen Abstraktem und Konkretem ohne Weiteres auf die Gegenüberstellung zwischen Verworrenheit und Deutlichkeit unserer Vorstellungen zurückzuführen. Wenn zwei Dinge unseren Sinnen anschaulich nur durch die wechselseitige Position als verschieden erscheinen, so nur, weil wir eine unpräzise Auffassung von ihnen haben; in Wirklichkeit dagegen sind sie immer verschieden aufgrund eines begrifflichen Merkmals, das ein Ding hat und das andere nicht. Diese Auffassung wurde von Kant auf Leibniz selbst übertragen. In der Kritik der reinen Vernunft, und zwar in der Amphibolie der Reflexionsbegriffe, in der Kant ausdrücklich auf die Leibniz'sche Tradition Bezug nimmt, ${ }^{30}$ schreibt er:

Er [i.e. Leibniz] verglich alle Dinge bloß durch Begriffe mit einander und fand, wie natürlich, keine andere Verschiedenheiten als die, durch welche der Verstand seine reinen Begriffe von einander unterscheidet. Die Bedingungen der sinnlichen Anschauung, die ihre eigene Unterschiede bei sich führen, sah er nicht für ursprünglich an; denn die Sinnlichkeit war ihm nur eine verworrene Vorstellungsart und kein besonderer Quell der Vorstellungen [...]. (KrV, B 326; meine Hervorhebungen)

Obwohl hier die komplexen historischen Beziehungen zwischen Kant, Leibniz und der Leibniz'schen Tradition nicht genau untersucht werden können, soll dennoch versucht werden, zumindest auf der systematischen Ebene nachzuweisen, dass Kants Unterscheidung zwischen „Begriff“ und „Anschauung“ auf das Problem zurückgeführt werden kann, das Leibniz selbst aufgestellt hatte, und das in der vorliegen Arbeit unter dem Stichwort Symmetrie bezeichnet wurde. Die Leibnizianer behaupteten, dass „diejenige Vorstellungsart, die wir bloße Anschauung nannten, eigentlich nur der verworrene Begriff von ihrem Gegenstande“ (AA 20: 278) sei, so dass jede Verschiedenheit immer eine Verschiedenheit von Begriffen sei. Für Kant gibt es dagegen eine Form von Verschiedenheit, die überhaupt nicht auf „Begriffe“, d.h. „allgemeine Vorstellungen“ (repraesentationes per notas communes) zurückgeführt werden kann, sondern nur in der „Anschauung“, d.h.

30 Vgl. dazu Funke, Gerhard: „Systematische Voraussetzungen der Leibniz-Kritik Kants im ,Amphibolie-Kapitel““. In: Akten des 4. Internationalen Kant-Kongresses. Hrsg. von Gerhard Funke. Berlin/New York 1974; Hess, Heinz-Jürgen: Zu Kants Leibniz-Kritik in der ,Amphibolie der Reflexionsbegriffe'. In: Beiträge zur Kritik der reinen Vernunft: 1781-1981; [Gerhard Lehmann zum 80. Geburtstag]. Hrsg. von Ingeborg Heidemann und Gerhard Lehmann. Berlin 1981, 201-232; Parkinson, George Henry R., Kant as a Critic of Leibniz: the Amphiboly of Concepts of Reflection. In: Revue Internationale de Philosophie 35, 1981, 302-314. 
in einer „einzelnen Vorstellung“ (repraesentatio singularis) gegeben werden kann (vgl. AA 09: 91). ${ }^{31}$

Begriffe sind verschieden, insofern in einem etwas ist, das in dem anderen nicht ist. Zwei geometrische Figuren können dagegen verschieden sein, wenn man sie zusammen in einer Anschauung vergleicht, obwohl nichts in einer zu finden ist, das in der anderen nicht ist. ${ }^{32}$ Nicht die Verschiedenheit der Dinge bewirkt die Verschiedenheit der Orte, wie die Leibnizianer meinten, sondern umgekehrt die bloße Verschiedenheit der Orte kann die Verschiedenheit der Dinge bewirken. ${ }^{33}$ Die vollkommene Homogenität des geometrischen Raumes ist also nicht die verworrene Manifestation der Verschiedenheit der Dinge, sondern es gibt umgekehrt eine Form von Verschiedenheit, die erst durch die gegenseitige Stellung im Raum von sonst vollkommen identischen Dingen hervortritt: „Wir schauen den Raum nicht an, aber auf verworrene Art; sondern er ist die Form unserer Anschauung “ (AA 23: 23; Refl. XXVIII). ${ }^{34}$

Diese Interpretationshypothese kann bestätigt werden, wenn man die Beispiele in Betracht zieht, von denen Kant ausgeht, um zu zeigen, dass der Raum kein „allgemeiner Begriff“ sein könne, sondern notwendigerweise als „reine Anschauung“ oder „Form der Anschauung“ angesehen werden müsse. Solche Beispiele scheinen dadurch verbunden zu sein, dass sie versuchen, die „Kongruenz“ geometrischer Figuren (die nur durch ihre wechselseitige Position oder Orientierung im Raum verschieden sind) von der logischen „Identität“ von Begriffen (die durch innere Eigenschaften voneinander abgegrenzt werden können) zu unterscheiden. Kant übernimmt von der Leibniz'schen Tradition die Idee, dass „die innern Bestimmungen eines Dinges [...] Qualitas und Quantitas“ (AA 28: 569s.) seien. Wenn zwei Begriffe dieselben inneren Bestimmungen haben, sind sie ein und derselbe Begriff (interne totaliter eadem non sunt diversa). Im Gegensatz dazu kann man zwei geometrische Figuren denken, die zwar in allen inneren Merkmalen übereinstimmen

31 Die Unterscheidung zwischen „Begriff“ und „Anschauung“ wurde besonders in der anglo-amerikanischen Debatte diskutiert. Vgl. dazu vor allem Hintikka, Jaakko: On Kant's Notion of Intuition (Anschauung). In: Logic, Language-Games and Information: Kantian Themes in the Philosophy of Logic. Hrsg. von T. Penelhum und J. J. Macintosh. Belmont, California 1969, 38-53; Howell, Robert: Intuition, Synthesis, and Individuation in the Critique of Pure Reason. In: Nô̂s 7/3, 1973, 207-232; Parsons, Charles: Kant's Philosophy of Arithmetic. In: Mathematics in Philosophy: Selected Essays. London 1983, 112. Die Grundpositionen in dieser Debatte sind in Smit, Houston: Kant on Marks and the Immediacy of Intuition. In: The Philosophical Review 109/2, 2000, 235-266: $237 \mathrm{f}$. gut zusammengefasst. Im deutschsprachigen Raum hat vor allem Gerhard Schönrich, die These hervorgehoben, dass die Anschauungsvorkommisse nur eine „Indexikalische“ Bedeutung haben. Vgl. Schönrich, Gerhard: Kant in der Diskussion der Moderne. Frankfurt am Main 1996, $105 \mathrm{f}$.

32 Vgl. Webb, Jeremy: Immanuel Kant and the Greater Glory of Geometry. In: Naturalistic Epistemology: A Symposium of Two Decades. Hrsg. von Abner Shimony und Debra Nails. Dordrecht/Boston/Norwell, MA 1987, 28.

33 „Die Verschiedenheit der <Dinge> Örter macht keine Verschiedenheit der Dinge selbst aus, sondern setzt sie voraus“ (AA 17:407, R. 4081); „Die Verschiedenheit der Örter beweiset die Verschiedenheit der Dinge“ (AA 17: 642, Refl 4673).

34 Vgl. dazu Calkins, Mary Whiton: Kant's Conception of the Leibniz Space and Time Doctrine. In: The Philosophical Review 6/4, 1897, 356-369. 
und demzufolge gleich und ähnlich sind, aber als verschieden betrachtet werden müssen, weil sie durch äußere Bestimmungen im Raum voneinander unterschieden werden können.

\subsection{Die inkongruenten Gegenstücke}

Das berühmteste, schon in der vorkritischen Zeit behandelte Beispiel, zu dem Kant greift, um die „anschauliche“ Natur des Raumes nachzuweisen, ist jenes der sogenannten „inkongruenten Gegenstücke“. ${ }^{35}$ Das Beispiel wurde, wie man weiß, zuerst in der vorkritischen Schrift Von dem ersten Grunde des Unterschiedes der Gegenden im Raume (1768) formuliert, um die Realität des absoluten Raumes nachzuweisen. Schon in der Dissertatio de mundis sensibilis atque intelligibilis

35 Vgl. dazu vor allem: The Philosophy of Right and Left. Incongruent Counterparts and the Nature of Space. Hrsg. von James Van Cleve und Robert E. Frederick. Dordrecht 1991. Nützlich sind außerdem der Anhang „Das Paradoxon symmetrischer Gegenstände“, in Vaihinger, Hans: Das Paradoxon symmetrischer Gegenstände. In: Kommentar zu Kants Kritik der reinen Vernunft. Hrsg. von Raymund Schmidt. Aalen 1970, Bd. 2, und der Aufsatz Scaravelli, Luigi: Gli incongruenti e la genesi dello spazio kantiano. In: Giornale critico della filosofia italiana 21, 1952, 389-421). An dieser Stelle soll es jedoch nicht um die Details des berühmten Kantischen Arguments und vor allem nicht um den Vergleich zwischen der vorkritischen und kritischen These Kants gehen (vgl. dazu z. B. Gloy, Karen: Die Kantische Differenz von Begriff und Anschauung und ihre Begründung. In: Kant Studien 75, 1984, 1-37). Es sei aber erlaubt, einige Bemerkungen darüber zu machen. Gemäß dem received view präsentierte Kant im Jahr 1768 ein Argument, das die Notwendigkeit eines Substantialismus im Gegensatz zum Relationalismus, anhand der Eigenschaft der Händigkeit demonstrieren sollte (vgl. z. B. Earman, John: Kant, Incongrous Counterparts and the Nature of Space and Space-Time. In: The Philosophy of Right and Left. Incongruent Counterparts and the Nature of Space. Hrsg. von James Van Cleve und Robert E. Frederick. Dordrecht 1991, 132). Meines Erachtens hat Kant aber statt der Gegenüberstellung zwischen Absolutem und Relativem eine andere Alternative als grundlegend betrachtet, die er in einer zwischen 1764-1766 geschriebenen Reflexion gut darstellt: „Entweder enthält der Raum den Grund der Möglichkeit von der compraesentz vieler substantzen und ihrer relationen, oder diese enthalten den Grund der Möglichkeit des Raumes“ (AA 17:293; Refl. 3790). Leibniz hätte behauptet, dass die Relationen der individuellen Substanzen (die prästabilierte Harmonie) die Basis für den Raum sind, für Kant soll dagegen gelten, dass die Relationen zwischen Dingen erst bestimmt werden können, wenn sie im Raum sind. Die Gegenüberstellung ist hier nicht Newton-Clarke gegen Leibniz, sondern etwa Leonhard Euler gegen die Wolffianer. Euler hatte nämlich die These vertreten, dass der Raum eine selbständige Existenz besitzt, weil der Ort keine Eigenschaft der Körper ist (vgl. Euler, Leonhard: Réflexions sur l'espace et le temps. In: Memoires de l'academie des sciences de Berlin 4, 1750, 324-33). Die Tatsache, dass Kant sich am Anfang der Schrift Von dem ersten Grunde des Unterschiedes der Gegenden im Raume (vgl. AA 02, 378) auf Euler beruft, ist nämlich mehr als bloß rhetorisch (vgl. Walford, David: The Aims and Method of Kant's 1768 Gegenden Im Raume Essay in the Light of Euler's 1748 Réflexions sur L'Espace“. In: British Journal for the History of Philosophy 7/2, 1999, 305-332): Was die vorkritische und kritische Raumlehre gemeinsam haben, scheint also die Behauptung des „Ganzheitscharakters“ des Raumes zu sein, die allen Lagenunterschieden vorangehen muss (vgl. dazu Gloy,Karen: Die Kantische Differenz von Begriff und Anschauung und ihre Begründung, 36). 
forma et principiis (1770; vgl. AA 02: 403), und später in den Prolegomena (1783; vgl. AA 04: 286) und in Metaphysische Anfangsgründe der Naturwissenschaft (1786, AA 04: 485) wurde es nochmals verwendet, hier jedoch um zu zeigen, dass Raum und Zeit „bloß[e] Formen unsrer sinnlichen Anschauung“ (AA 04: 285) sind. Wir werden nur auf diese reifere Version des Arguments Bezug nehmen.

Mit diesem Beispiel versucht Kant zu zeigen, dass das, was logisch die Kriterien für die Identität von Begriffen erfüllt, als geometrisch verschieden betrachtet werden kann. ${ }^{36}$ Es geht, wie man weiß, um eine körperliche Figur und ihr Spiegelbild, die gleich und ähnlich und trotzdem nicht kongruent sind, weil sie in einem dreidimensionalen Raum nicht miteinander zur Deckung gebracht werden können: „aequalia et similia congruunt non nisi in plano“(AA 18: 15). Eine ein- oder zweidimensionale Figur kann immer mit ihrem Spiegelbild durch eine Drehung in der zweiten oder in der dritten Dimension zur Deckung gebracht werden; im Fall einer dreidimensionalen Figur hat man dagegen keine vierte Dimension, die eine solche Bewegung ermöglicht. ${ }^{37}$

Zwei Begriffe können durch bestimmte Merkmale unterschieden werden, die ein Begriff besitzt und der andere nicht. Zwei geometrische Körper wie die linke und die rechte Hand können dagegen völlig gleich und ähnlich sein, wenn man die inneren Bestimmungen (Quantität und Qualität) betrachtet, und trotzdem so verschieden wegen ihrer äußeren Verhältnisse, dass „der Handschuh der einen Hand [...] nicht auf der andern gebraucht werden “ (AA 04: 286) kann. Denn es ist unmöglich zu zeigen, welche Eigenschaft eine rechtsorientierte Figur besitzt, die sie von einer linksorientierten unterscheidet, weil „eine vollständige Beschreibung der einen in allen Stücken auch von der andern gelten " muss (AA 02: 382). So sind sie ähnlich und gleich, „wenn er allein und zugleich vollständig beschrieben wird“ (AA 04: 286); ${ }^{38}$ sie sind aber verschieden, wenn man beide Figuren zusammen anschaut, da man damit ihre wechselseitigen Verhältnisse betrachten kann. Es gibt

36 „L'égalité géométrique ne se réduit pas à l’identité logique“ (Vuillemin, Jules: La théorie kantienne de l'espace à la lumière de la théorie des groupes des transformations. In: Mind 51/3, 1967, 332-351: 332).

37 Vgl. dazu Möbius, August Ferdinand: Der Barycentrische Calcul. Ein neues Hülfsmittel zur analytischen Behandlung der Geometrie. In: Gesammelte Werke. Hrsg. von Felix Klein et al. Leipzig 1885, Bd. 1, 171; Mongré, Paul (Felix Hausdorff): Das Chaos in kosmischer Auslese. Ein erkenntnisskritischer Versuch. Leipzig 1898, 91. Neudruck in Hausdorff, Felix: Das Chaos in kosmischer Auslese. Ein erkenntnisskritischer Versuch. In: Gesammelte Werke. Hrsg. von Werner Stegmaier. Berlin 2006, Bd. VII. So auch Ludwig Wittgenstein: „Das Kant'sche Problem von der rechten und linken Hand, die man nicht zur Deckung bringen kann, besteht schon in der Ebene, ja im eindimensionalen Raum, wo die beiden kongruenten Figuren a und $\mathrm{b}$ auch nicht zur Deckung gebracht werden können, ohne aus diesem Raum herausbewegt zu werden. [...]. Den rechten Handschuh könnte man an die linke Hand ziehen, wenn man ihn im vier-dimensionalen Raum umdrehen könnte." (Wittgenstein, Ludwig: Tractatus logico philosophicus. Logisch-philosophische Abhandlung. Frankfurt am Main 2003, 105: 6.362; vgl. dazu Bazzocchi, Luciano „Il codice segreto di Wittgenstein. Dagli opposti incongruenti di Kant al luogo logico della proposizione negante. In: Studi kantiani 19, 2006, 131-140: 132).

38 „[W]enn man bloß auf eine derselben allein sieht“ (AA 02:382; meine Hervorhebung). 
also eine durch begriffliche Merkmale nicht ausdrückbare Art von Verschiedenheit, die nur durch einen anschaulichen Vergleich gegeben werden kann:

Wir können daher auch den Unterschied ähnlicher und gleicher, aber doch incongruenter Dinge [...] durch keinen einzigen Begriff verständlich machen, sondern nur durch das Verhältniß zur rechten und linken Hand, welches unmittelbar auf Anschauung geht. (AA 04: 286; meine Hervorhebung)

Man kann zwar erkennen, dass ein Ding das Spiegelbild des anderen ist. Doch ist die Erklärung unvollständig, denn sie sagt nicht, welches das rechte und welches das linke Ding ist. Das einzige Mittel ist, den Unterschied zu zeigen, d.h., wie Kant auch sagt, auf „das Gefühl eines Unterschiedes an meinem eigenen Subject, nämlich der rechten und linken Hand“ zurückzugreifen (AA 08: 135). ${ }^{39}$

Im Gegensatz zu seiner vorkritischen Meinung (vgl. GP, AA 02: 382), scheint Kant hier also zu behaupten, dass, wenn es im gesamten Universum nur eine Schraube gäbe, man nicht sagen könnte, ob es sich um eine linksdrehende oder um eine rechtsdrehende handelt. ${ }^{40}$ Diese Frage hat nämlich gar keine Bedeutung: Was eine rechte Schraube ist, wird durch ihre Lage bestimmt, z. B. hinsichtlich einer linken Schraube oder wenn man so will, hinsichtlich irgendeines asymmetrischen Gegenstandes, etwa auch eines als rechts dekretierten Achsensystems. ${ }^{41}$ So hätte es auch keinen Sinn, sich zu fragen, ob ein einzelner Körper im Universum in Ruhe

39 Vgl. Borel, Émile: Zeit und Raum. Von Euklid bis Einstein. Stuttgart 1931, 68-71. Für den Vergleich mit Leibniz vgl. De Risi: Geometry and Monadology: Leibniz's analysis situs and Philosophy of Space, $291 \mathrm{f}$. Man muss nämlich zwischen den folgenden beiden Fällen unterscheiden: „a) Das Phänomen der Händigkeit als solcher im Sinne einer ,gleichwertigen Existenz rechts- und linkshändiger Objekte (symmetrischer Fall). b) Die Auszeichnung einer bestimmten Händigkeit im Sinne einer, Which-is-which'-Frage (asymmetrischer Fall) “. (Lyre, Holger: Metaphysik im ,Handumdrehen': Kant und Earman, Parität und moderne Raumauffassung. In: Philosophia naturalis 42/1, 2005, 49-76: 62). Meines Erachtens bezieht sich Kant normalerweise auf das zweite Problem, wenn er behauptet, dass der „Unterschied“ zwischen rechts und links nur in der Anschauung gegeben werden kann. Es wurde eben erläutert, dass das auch das Problem Leibniz' ist. Auch Carl F. Gauß benutzt den Ausdruck „Unterschied“ zwischen links und rechts in diesem Sinn. S. u. Anm. 43.

40 Der „vorkritische“ Kant vertrat, wie man weiß, die entgegengesetzte These: „[D]as erste Schöpfungsstück solle eine Menschenhand sein, so ist es nothwendig entweder eine Rechte oder eine Linke" (AA 02: 382s.). Auch in diesem Fall ist es merkwürdig, dass schon Euler ein ähnliches Beispiel lieferte: „Eben so würde man, wenn nur ein einziger Körper existirte, behaupten müssen, dass derselbe entweder ruhe oder sich bewege, da man eben so wenig beides zugleich, als weder das eine noch das andere aufstellen kann “. Da das unmöglich ist, sollte man den Körper in Bezug auf den absoluten Raum betrachten (Euler, Leonhard: Leonhard Euler's Theorie der Bewegung fester oder starrer Körper. Greifswald 1853, 36 f.). Da der „kritische“ Kant die vollkommene Relativität des Raumes angenommen hat, scheint es aber berechtigt zu vermuten, dass er seine vorherige Meinung nicht mehr vertreten konnte: eine einzige Hand im Universum wäre weder linksorientiert noch rechtsorientiert, so wie ein einziger Körper im Universum weder in Ruhe noch in Bewegung wäre: „Daß [keine] (g alle) relation im Raume [einseitig] (g relativisch und niemals absolut) sey; z.E. kein absoluter Ort, keine absolute ruhe oder Bewegung, sondern iederzeit Bewegung und ruhe sich auf irgend einen andern Korper beziehen müsse" (AA 17: 453; Refl. 4200).

41 Vgl. Remnant, Peter: Incongruent Counterparts and Absolute Space. In: Mind 72/287, 1963, 393-399: 393; Freudenthal, Hans: Der Orientierte Raum des Mathematikers. In: Die Naturwissenschaften 50/6, 1963, 199-205: 202. 
oder in Bewegung ist, weil Ruhe und Bewegung nur in Bezug auf andere Körper definiert werden können; so ist „z.B. Bewegung oder Ruhe der Welt im unendlichen leeren Raum, eine Bestimmung des Verhältnisses beider untereinander, welche niemals wahrgenommen werden kann und also auch das Prädicat eines bloßen Gedankendinges ist" ( $\mathrm{KrV}, \mathrm{B} 457, \mathrm{Fn})$.

Zwei Schrauben von derselben Form und Größe besitzen also eine bestimmte Händigkeit, eine ist linksorientiert und die andere rechtsorientiert, dies hängt jedoch nicht von den Eigenschaften ab, die eine besitzt und die andere nicht, sondern von den Relationen zu einem dritten Körper, der als rechts- oder linksdrehend dekretiert wird. Der Unterschied zwischen einer rechten Schraube ist also für Kant doch „innerlich“ (eine rechte Schraube können wir bewegen, wie wir wollen, es wird aber niemals eine linke daraus), aber kein „innerer“, weil es keine Eigenschaft gibt, die eine linksorientierte Schraube besitzt und eine rechtsorientierte nicht. ${ }^{42}$ Keine noch so vollständige begriffliche Beschreibung einer Schraube kann feststellen, ob sie links- oder rechtsdrehend ist, sondern es erfordert einen Akt willkürlicher Wahl, d.h. direkter anschaulicher Aufweisung eines einzelnen Objekts, um festzustellen, ob es links- oder rechtsorientiert ist. Nachdem die Wahl jedoch für einen Körper getroffen ist, ist sie für jeden Körper festgelegt. Man kann also nicht „durch Worte“ erklären, was rechts und links heißt, sondern muss es „durch Hinweis“ zeigen. ${ }^{43}$

42 „Nun sind hier keine innre Unterschiede, die irgend ein Verstand nur denken könnte; und dennoch sind die Unterschiede innerlich, so weit die Sinne lehren, denn die linke Hand kann mit der rechten unerachtet aller beiderseitigen Gleichheit und Ähnlichkeit doch nicht zwischen denselben Grenzen eingeschlossen sein (sie können nicht congruiren); der Handschuh der einen Hand kann nicht auf der anderen gebraucht werden." (Prol, AA 04: 286) An anderer Stelle benutzt Kant aber „inner“ und „innerlich“ mit gerade umgekehrter Bedeutung: „und hier ist denn doch eine innere Verschiedenheit beider Triangel, die kein Verstand als innerlich angeben kann, und die sich nur durch das äußere Verhältniß im Raume offenbart" (Ebenda).

43 Dieses Problem wird am deutlichsten von Carl Friedrich Gauß erläutert: „Der Unterschied zwischen Rechts und Links läßt sich aber nicht definieren, sondern nur vorzeigen, so daß es damit eine ähnliche Bewandtnis hat, wie mit Süß und Bitter." Zwei Individuen „können sich über Rechts und Links nicht anders unmittelbar verständigen, als indem ein und dasselbe materielle individuelle Ding eine Brücke zwischen ihnen schlägt" (Gauß, Carl Friedrich: Werke. Hrsg. von der Königl. Ges. d. Wiss. zu Göttingen, 12 Bde. Hildesheim/New York. 1973, VIII, 247, meine Hervorhebung). An anderer Stelle schreibt Gauß, dass „Dieser Unterschied zwischen rechts und links [...] nur durch Nachweisung an wirklich vorhandenen materiellen Dingen" mitgeteilt werden könnte" (a.a.O., Bd. II, 177; meine Hervorhebung). Obwohl Gauß diese Bemerkung gegen Kant formulierte, ist in vorliegendem Zusammenhang entscheidend, dass er ausdrücklich erkannt hat, dass der Unterschied zwischen rechts und links nicht auf Begriffe zurückgeführt werden kann: „Diesen Unterschied [...] kann man aber nicht auf Begriffe bringen, sondern nur aus dem Anhalten an wirklich vorhandene räumliche Dinge vorzeigen" (a.a.O., 7:248; meine Hervorhebung). Vgl. dazu Timerding, Heinrich Emil: Kant und Gauß. In: Kant-Studien 28, 1923, 34-37 und Vaihinger: Das Paradoxon symmetrischer Gegenstände, 527. Vgl. auch Bennett, Jonathan: The Difference between Right and Left. In: The Philosophy of Right and Left. Incongruent Counterparts and the Nature of Space. Hrsg. von James Van Cleve und Robert E. Frederick. Dordrecht 1991, 97-130: 100, 09. Auch De Risi scheint auf dieses Problem die Übereinstimmung zwischen Leibniz und Kant zurückzuführen (vgl. De Risi: Geometry and Monadology: Leibniz's analysis situs and Philosophy of Space, 291). 


\subsection{Positive und negative Größen}

Kant zufolge hat also die deutsche Schulmetaphysik des 17. Jahrhunderts logische Identität und geometrische Kongruenz verwechselt. Die Inkongruenz der Gegenstücke, d.h. ihre wechselseitige „Unersetzbarkeit“, zeigt für Kant, dass dasjenige, was für die „Logik der Begriffe“ identisch ist, vollkommen verschieden bleibt für die „Logik der Anschauung“. Kant hat hier Leibniz’ ErsetzbarkeitsGedanken vollkommen missverstanden, ${ }^{44}$ in dem Sinne, als ob er die Negierung jeder reellen Differenz wäre, die Feststellung einer absoluten Identität. Der Verdienst Kants scheint aber darin zu bestehen, dass er in Gegensatz zu Leibniz bemerkte, dass eine Kongruenz entweder eigentlich (sie führt eine links- bzw. rechtsgewundene Schraube in eine ebensolche über), oder uneigentlich (sie verwandelt eine linksgewundene Schraube in eine rechtsgewundene und umgekehrt) sein kann. Die erste wird einfach „Bewegung“ genannt (in einem geometrischen Sinn), während die letzte als „Spiegelungen“ bekannt ist. ${ }^{45}$ Kant schreibt einer solchen

44 Man kann vermuten, dass diese Missdeutung auf die Wolffsche Darstellung zurückzuführen ist. Wolff definierte nämlich beide, logische Identität und Kongruenz, durch das Salva-veritate-Kriterium. In seiner Philosophia prima sive Ontologia definiert Christian Wolff die logische Identität wie folgt: „Eadem dicuntur, quae sibi substitui possunt salvo quocumque pradicato" (Wolff, Christian: Philosophia prima sive Ontologia (1730). In: Ders., Gesammelte Werke. Hrsg. von Jean Ecole, 30 Bde., Hildesheim 1962, Bd. II.3, $\$ 181$ ). Die Kongruenz wird ähnlich charakterisiert: „res congruentes prorsus eadem sunt, consequenter unam alteri substituere licet salvo omni praedicato“, z. B. „si duo triangula congruunt, unum alteri eo successu substituere licet, ut non appareat, substitutionem fuisse facta" (a.a.O. $\$ 467)$.

45 Die These einiger Interpreten, dass das von Kant hervorgehobene Problem der Inkongruenz der Gegenstücke einfach eine „kombinatorische Grundlage“ (Mühlhölzer, Felix: Das Phänomen der Inkongruenten Gegenstücke aus Kantischer und heutiger Sicht. In: Kant Studien 83/4, 1992, 436-453: 449-451) hätte, und deswegen rein „begrifflich“ wäre (vgl. dazu auch Reidemeister, Kurt: Über den Unterschied der Gegenden im Raum. In: Zeitschrift für philosophische Forschung 2, 1947, 131-150 und Reidemeister: Raum und Zahl, 70-99), trifft meines Erachtens das Grundproblem Kants nicht. Mühlhölzer beruft sich auf die Autorität Weyls, der in der Philosophie der Mathematik und Naturwissenschaft vom gruppentheoretischen Standpunkt ausgehend, schreibt: „Das Phänomen, über das sich Kant wundert, kann somit höchst befriedigend unter allgemeine und abstrakte ,Begriffe" gestellt werden“ (Weyl: Philosophie der Mathematik und Naturwissenschaft, 108. In der 1928 als Beitrag zu dem von Alfred Bäumler und Manfred Schröter herausgegebenen Handbuch der Philosophie erschienenen Auflage des Buches, schrieb aber Weyl noch dazu: „Die erkenntnistheoretische Bedeutung des Relativitätsproblems [...] soll damit nicht geleugnet werden“, d.h. die These, dass „die Relation ,widersinnig gewunden' nicht in einer für sich heraushebbaren Eigenschaft des einen und des andern Relationsgliedes fundiert ist." (Weyl, Hermann: Philosophie der Mathematik und Naturwissenschaft. In: Handbuch der Philosophie. Hrsg. von Alfred Bäumler und Manfred Schröter. München/Berlin 1928, 60). Die Existenz rechts- und linksorientierter Koordinatensysteme ist sicherlich rein „begrifflich“, aber, wie auch Weyl behauptet, kann die Frage, welche ist links, welche ist rechts, nur durch den Bezug auf „einen unmittelbar aufgewiesenen Gegenstand“, nämlich „dies da“ anschaulich beantwortet werden (Weyl: Philosophie der Mathematik und Naturwissenschaft, 21). Vgl. auch Anm. 39 der vorliegenden Arbeit. 
„Entdeckung“, ${ }^{46}$ eine relevante philosophische Bedeutung zu; sie zeigt nämlich, dass logische Identität und geometrische Kongruenz nicht verwechselt werden können. ${ }^{47}$ Bei bloßer Bewegung im Raum könnte man immer noch sagen, dass „dasselbe“ Objekt gedreht oder verschoben wurde. Will man dagegen das materielle Spiegelbild eines nicht spiegelsymmetrischen Körpers vor sich haben, muss man ihn, so zu sagen, neu bauen. Urbild und Abbild sind also sicher nicht „dasselbe“; es ist aber unmöglich, im Einen irgendeine Eigenschaft zu finden, die im Anderem nicht zu finden ist. Kant hat damit den Äquivalenzbegriff wieder gewonnen: Links und rechts sind doch gleichberechtig, aber nicht identisch im logischen Sinne, weil sie doch verschieden in Bezug aufeinander bleiben.

Die Inkongruenz ist hier also nicht entscheidend. ${ }^{48} \mathrm{Wichtig}$ ist vielmehr die Verschiedenheit der „Orientierung“ (oder, wie Kant normalerweise schreibt, der „Richtung“). ${ }^{49}$ Dies kann bestätigt werden, wenn man ein anderes, schon in der vorkritischen Zeit (nämlich in der Schrift Versuch den Begriff der negativen Größen in die Weltweisheit einzuführen, 1763) besprochenes Problem der Unterscheidung von positiven und negativen Größen in Betracht zieht. Eine „Richtung“ (Sinn) kann nicht für sich von der anderen unterschieden werden. Welche Richtung man „nach links“ und „nach rechts“ nennen soll, ist durch den begrifflich zu beschreibenden Charakter der Linie nicht gegeben; „ $+a$ und $-a$ sind einander nicht qualitativ, sondern nur in der Relation der Richtung entgegengesetzt “ (OP, AA 22: 176). Die Richtungen (Sinne) können also per se nicht unterschieden werden, und die Entgegensetzung tritt erst auf, wenn man sie anschaulich vergleicht: „Allein in

46 Vgl. dazu: Das Problem wurde schon von Legendre bemerkt Hon, Giora und Bernard R. Goldstein: Kant vs. Legendre on Symmetry. Mirror Images in Philosophy and Mathematics. In: Centaurus, 47/4, 2005, 283-297.

47 Vgl. dazu z.B. Guarducci, Alfredo: Della congruenza o del movimento. In: Questioni riguardanti la geometria elementare. Hrsg. von Federigo Enriques und Ugo Amaldi. Bologna 1900, 65-82: 65.

48 Von diesem Standpunkt aus ist die oft gemachte Bemerkung, dass die von Kant behauptete Inkongruenz nur lokal und zufällig ist, denn, wenn der Raum global nicht orientierbar wäre (etwa wie im sogenannten „Möbiusband“), könnten die Gegenstücke, z.B. nach eine Reise in einen weiteren Raum, beiden zur Deckung gebracht werden (Vgl. Nerlich, Graham: Hands, Knees, and Absolute Space. In: The Philosophy of Right and Left Incongruent Counterparts and the Nature of Space. Hrsg. von James Van Cleve und Robert E. Frederick. Dordrecht 1991). Diese Bemerkung, obwohl sehr nützlich um die Natur von Kants Paradoxon zu verstehen, trifft meines Erachtens nicht das Ziel des Kantischen Arguments, das vielmehr auf die nur, ,indexikalisch vollziehbaren Unterscheidungen von linker und rechter Hand bzw. ihrer Spiegelbilder “ gerichtet ist (Schönrich, Gerhard: Das normative Dilemma in Kants Erkenntnistheorie. In: Normativität und Faktizität. Skeptische und transzendentalphilosophische Positionen im Anschluss an Kant. Hrsg. von Gerhard Schönrich. Dresden 2004, 67-86: 82). Was man links und rechts nennt, ist willkürlich. Hat man sich aber einmal entschieden, so ist der Raum orientiert.

49 In der heutigen Ausdrucksweise sagt man, dass alle parallelen Vektoren dieselbe „Richtung “ haben; parallele Vektoren können aber entgegengesetzte „Orientierungen“ darstellen. Kant benutzt in beiden Fällen das Wort „Richtung“ und, wie in den Metaphysischen Anfangsgründen der Naturwissenschaft, unterscheidet er zwischen beiden Bedeutungen des Wortes (vgl. MAN, AA 04:483). An einer Stelle der Danziger Physik dagegen unterscheidet er zwischen „Direktion“ und „Gegend“ (vgl. Danziger Physik, AA 29: 113). 
der sinnlichen Anschauung, darin Realität (z. B. Bewegung) gegeben wird, finden sich Bedingungen (entgegengesetzte Richtungen), von denen im Begriffe der Bewegung überhaupt abstrahirt war" (KrV, B 338; meine Hervorhebungen), da man „entgegenstehende Richtungen [...] nur in der Anschauung, nicht in bloßen Begriffen vorstellen " (Entwurf FM, AA 20: 283; meine Hervorhebungen) kann. ${ }^{50}$

In Metaphysische Anfangsgründe der Naturwissenschaft (1786) verbindet Kant ausdrücklich das Problem der inkongruenten Gegenstücke mit dem Problem der Bewegungen in entgegengesetzten Richtungen oder Sinnen. Betrachtet man z. B. eine Kreisbewegung, kann man sich fragen:

[W] as ist hier die Seite, nach der die Bewegung gerichtet ist? eine Frage, die mit der eine Verwandtschaft hat: worauf beruht der innere Unterschied der Schnecken, die sonst ähnlich und sogar gleich, aber davon eine Species rechts, die andere links gewunden ist [...] ein Begriff, der sich zwar construiren, aber als Begriff für sich durch allgemeine Merkmale und in der discursiven Erkenntnißart gar nicht deutlich machen läßt [...]. (MAN, AA 04: 483-484)

In beiden Fällen geht es um Gegenstände, die nicht zu unterscheiden sind, wenn sie isoliert betrachtet werden, denn es ist unmöglich, „in der Beschreibung eines Cirkels, ohne an ihm irgend eine Verschiedenheit der Gegenstände zu bedürfen, doch die Bewegung von der Linken zur Rechten von der in entgegengesetzter Richtung zu unterscheiden“ (WDO, AA 08: 135). Das, was in einem gegebenen Raum nach der einen Seite zu, und das, was nach der anderen zu liegt, kann bei aller Schärfe des Verstandes nicht begrifflich beschrieben, d.h. auf Verstandesmerkmale zurückgeführt (vgl. MSI, AA 02: 403), sondern nur direkt anschaulich aufgewiesen werden, weil „sich dieser Unterschied zwar in der Anschauung geben, aber gar nicht auf deutliche Begriffe bringen, mithin nicht verständlich erklären (dari, non intelligi) läßt" (MAN, AA 04: 484, meine Hervorhebung).

Wie Leibniz erwähnt deswegen auch Kant in Was heißt: Sich im Denken orientiren? (1786) das Gedankenexperiment der Spiegelung des ganzen Universums. Die gespiegelte Welt wäre von der ursprünglichen nicht zu unterscheiden, solange alle Gegenstände die gleiche Vertauschung erleiden: ${ }^{51}$

$[U] n d$ wenn in einem Tage durch ein Wunder alle Sternbilder zwar übrigens dieselbe Gestalt und eben dieselbe Stellung gegen einander behielten, nur daß die Richtung derselben, die sonst östlich war, jetzt westlich geworden wäre, so würde in der nächsten sternhellen Nacht zwar kein menschliches Auge die geringste Veränderung bemerken. (WDO, AA 08: 135)

Die zwei „Sinne“ sind nämlich indifferent; wenn nämlich die Planeten sich von rechts nach links, für einen Beobachter auf der Sonne mit dem Kopf nach Norden und den Füßen nach Süden, bewegen, drehen sie sich jedoch von links nach rechts für einen Beobachter, der sich in der umgekehrten Position befindet. Die Unterscheidung zwischen den zweien „Sinnen“ ist relativ zu jener zwischen Norden und Süden, die aber auch relativ ist. Auch dieses sehr „Leibnizsche“ Beispiel zeigt also,

50 Die Verbindung zwischen dem Unterschied der inkongruenten Gegenstücke und jenem der positiven und negativen Größen und die Tatsache, dass beide für Kant nur in der Anschauung gegeben werden können wurde, soweit ich sehen kann, in der Literatur nicht bemerkt. Vgl. aber Russell, Bertrand: The Principles of Mathematics. Cambridge 1903, 227.

51 Vgl. Mongré: Das Chaos in kosmischer Auslese. Ein erkenntnisskritischer Versuch, 89. 
wie es in der Paradoxie der inkongruenten Gegenstücke vor allem um die Unmöglichkeit geht, die „Orientierung“ begrifflich zu definieren, ein Raumgebilde von seinem Spiegelbild durch „innere Kriterien“ zu unterscheiden:52 „Daß sich alle Planeten u. ihre Trabanten von Abend gegen Morgen fortschreitend sowohl als umdrehend bewegen “, ist also nach Kant eng verbunden mit „dem Phänomen der rechten und linken Hand“ (OP, AA 22: 535).

\subsection{Verschiedenheit des Ununterscheidbaren}

Der Bezug zu Leibniz tritt besonders deutlich hervor, wenn man ein anderes, vor allem in der Kritik der reinen Vernunft, im Anhang Von der Amphibolie der Reflexionsbegriffe, diskutiertes Beispiel betrachtet, das gegen „Leibnitzens Satz des Nichtzuunterscheidenden“ (AA 20: 280) gerichtet ist: „Es ist die frage, ob 2 Dinge blos numero verschieden seyn können oder ob sie durchaus specifisch plura seyn müssen, um mehr als ein Ding zu seyn" (AA 17: 683; Refl. 4712). ${ }^{53}$

Dieses Problem, (dass - soweit ich sehen kann - in der Sekundärliteratur nicht bemerkt wurde), hat eine evidente Ähnlichkeit mit jenem der inkongruenten Gegenstücke. Es geht nämlich nochmals um zwei Objekte, die gleich und ähnlich und trotzdem wegen ihrer wechselseitigen Position im Raum verschieden sind: „Wenn uns ein Gegenstand mehrmals, jedesmal aber mit eben denselben innern Bestimmungen (qualitas et quantitas) dargestellt wird, so ist derselbe, wenn er als Gegenstand des reinen Verstandes gilt, immer eben derselbe und nicht viel, sondern nur ein Ding (numerica identitas) “ (KrV, B 319); wenn der Gegenstand aber nicht durch „Begriffe“, sondern in der „Anschauung“ gegeben ist, „ist doch die Verschiedenheit der Örter“, nämlich eine äußere Relation, „ein genugsamer Grund der numerischen Verschiedenheit [numerica diversitas] des Gegenstandes (der Sinne) selbst“ (KrV, B 319). Hier ein Beispiel Kants:

Der Begriff von einem Kubikfuße Raum, ich mag mir diesen denken, wo und wie oft ich wolle, ist an sich völlig einerlei. Allein zwei Kubikfüße sind im Raume dennoch bloß durch ihre Örter unterschieden (numero diversa); diese sind Bedingungen der Anschauung, worin das Object dieses Begriffs gegeben wird, die nicht zum Begriffe, aber doch zur ganzen Sinnlichkeit gehören. (KrV, B 338; meine Hervorhebungen)

Wenn „A und B [...] in Ansehung aller ihrer innern Bestimmungen (der Qualität und Quantität) völlig einerley sind“, sind sie ein und derselbe Begriff; in der Anschauung dagegen können sie „doch durch die Örter im Raume“ unterschieden werden, „weil ganz ähnliche und gleiche Räume außer einander vorgestellt werden können“ (AA 20: 282).

Man kann also letztlich behaupten, dass alle bisher betrachteten Beispiele Kants, um Begriff und Anschauung zu unterscheiden (die inkongruenten Gegenstücke, die positiven und negativen Größen, die zwei Kubikfuß an verschiedenen

52 Vgl. ebenda.

53 Herring, Herbert: Leibniz' principium identitatis indiscernibilium und die Leibniz-Kritik Kants. In: Kant Studien 69, 1957, 389-400; vgl. auch PND, AA 01: 409. 
Orten), auch wenn sie nicht immer von Kant selbst in Verbindung gebracht werden, etwas gemein haben. Es geht um Elemente, die dieselben inneren Merkmale (notae internae), Quantität und Qualität, besitzen, d.h. gleich und ähnlich, und trotzdem durch äußere wechselseitige Relationen (ratione relationis; AA 29: 838) verschieden sind, d.h. nur wenn sie in der Anschauung verglichen werden. ${ }^{54}$ Wenn dagegen die Dinge nur durch Begriffe als Noumena betrachtet werden, ist es immer möglich, innere Unterscheidungen zu finden, die ein Ding besitzt und das andere nicht. Man könnte also behaupten, dass für Kant die Welt der Dinge an sich vollkommen asymmetrisch ist, während nur die Erscheinungen gewisse Symmetrien darstellen, die ihre naturwissenschaftliche Erkenntnis ermöglichen.

\subsection{Der Raum als reine Anschauung}

Wenn also Kant den Raum der „Logik der Anschauung“ und nicht der „Logik des Begriffes“ unterwirft, bedeutet das zunächst, dass ein Teil des Raumes für sich betrachtet von irgendeinem anderen gar nicht zu unterscheiden ist; man unterscheidet sie erst, wenn mehrere Teile in einer Anschauung gleichzeitig aufgefasst werden:

Denn ein Theil des Raums, ob er zwar einem andern völlig ähnlich und gleich sein mag, ist doch außer ihm und eben dadurch ein vom ersteren verschiedener Theil [...]; und dieses muß daher von allem, was in den mancherlei Stellen des Raums zugleich ist, gelten, so sehr es sich sonst auch ähnlich und gleich sein mag. (KrV, B 320; meine Hervorhebungen)

Durch die Unterscheidung zwischen Begriff und Anschauung versucht Kant also, eine von moderner mathematischer Naturwissenschaft festgestellte Eigenschaft des Raumes „erkenntnistheoretisch“ zu begründen, d.h. mit den Worten Leonhard Eulers, „que tout le different lieu ou partie de l'espace soient semblable entre eux" [alle verschiedenen Orte oder Teile des Raumes sind einander ähnlich]. ${ }^{55}$ Ein geometrischer Punkt für sich betrachtet ist von irgendeinem anderen

54 So Johan Schultz: „Die innern Merkmale eines Dinges heißen diejenigen, die seine Qualität und Quantität betreffen, die äußern die welche seine Stelle im Raum und in der Zeit bezeichnen“; „Dinge, die sich durch kein inneres Merkmal unterscheiden lassen, heißen an sich betrachtet vollkommen einerley [...] Also können Dinge, die an sich betrachtet vollkommen einerley sind, bloß in Ansehung ihrer Stelle im Raum und in der Zeit verschieden seyn " (Schultz, Johann: Anfangsgründe der reinen Mathesis. Königsberg 1790, 28). Vgl. dagegen Salomon Maimon, der die Position der „Leibnitianer“ verteidigte: „Ich würde aber hierauf statt Leibniz erwiedern, daß nach [...] die Verschiedenheit der äußeren Verhältnisse in Zeit und Raum in der Verschiedenheit der innern Beschaffenheit gegründet seyn muß.“ Maimon, Salomon: Versuch einer neuen Logik oder Theorie des Denkens. Nebst angehängten Briefen des Philaletes an Änesidemus. In: Ders.: Gesammelte Werke. Hrsg. von Valerio Verra, 7 Bde., Hildesheim 1965, Bd. 5, 198; Leibniz hielt nämlich „die Erscheinungen für Dinge an sich selbst, in deren Verstellung die Sinnlichkeit eine gewisse Verworrenheit verursacht, die der Verstand durch Entwicklung ihrer Begriffe wegschaffen muß.“ Maimon also behauptet wie Leibniz, dass „es in der Natur nicht zwei völlig ähnliche und gleiche Dinge geben kann, obgleich die Sinnlichkeit es zu glauben verleitet" (a.a.O., 193).

55 Euler: Réflexions sur l'espace et le temps, 330. 
gar nicht zu unterscheiden, d.h. nach der Definition, die wir schon bei Leibniz gefunden haben, sind alle Punkte in einem „einigen, allbefassenden Raum“:

Der Raum ist kein discursiver oder, wie man sagt, allgemeiner Begriff [...], sondern eine reine Anschauung. Denn erstlich kann man sich nur einen einigen Raum vorstellen, und wenn man von vielen Räumen redet, so versteht man darunter nur Theile eines und desselben alleinigen Raumes. (KrV, B 39)

Während die Begriffe sich hierarchisch strukturieren (nach Arten und Gattungen), sind alle Teile des Raumes vollkommen gleichberechtigt. Statt der Identität der traditionellen Logik tritt hier eine bloße Äquivalenz auf. Der Anschauungsbegriff drückt also einfach jene „Einheit des Raumes“ aus, die von Leibniz selbst, wie wir sahen, zum Ausgangspunkt seiner Lehre gemacht worden ist ${ }^{56}$ Dass der Raum eine reine Anschauung oder Form der Anschaunng ist, bedeutet also nur der Grundsatz der durchgängigen Homogenität des Raumes, ${ }^{57}$ die Indifferenz der geometrischen Gebilde aller Lageunterschiede und damit die prinzipielle Bestimmungs- und Eigenschaftslosigkeit der Teile des Raumes. Der Raum kann nämlich willkürlich in Teile eingeteilt werden, die (entweder direkt durch Deckung oder nur indirekt durch Spiegelung) alle gleich und ähnlich sind und die lediglich durch das Verhältnis zu einem einzelnen, empirisch gegebenen Bezugsobjekt von den anderen unterschieden werden können. ${ }^{58}$

Die Auffassung des Raumes „als von der Erfahrung abstrahirte, obzwar in der Absonderung verworren vorgestellte Verhältnisse der Erscheinungen “ (KrV, B 57-58), ist also das, was Kant von dem Raumbegriff der Leibnizianer ablehnt.

56 Lazurus Bendavid, einer der ersten Vertreter der kantischen Philosophie, hat meines Erachtens diesen Standpunkt sehr deutlich ausgedrückt: „Zur Bildung eines allgemeinen Begriffes gehören bekanntermaßen, mehrere, in etwas gleiche und in etwas verschiedene Dinge, von denen man ihre individuelle Verschiedenheit weglässt und nur das behält, was ihnen gemeinschaftlich zukommt. [...]. Nun aber findet zwischen Raum und Raum gar kein Unterschied statt, und die Vorstellung desselben kann kein allgemeiner Betriff seyn. Wenn ich den Raum meines Zimmers von dem des Nebenzimmers unterscheide, so geschieht das, weil beyde Zimmer durch eine Wand getrennt werden. Nähme ich diese Wand weg, so wären beyde, vorhin verschiedene Räume, nur ein Raum; und eben so würde es durch Aufhebung aller Schranken eines bestimmten Raumes, überall nur einen Raum geben." Bendavid, Lazarus: Vorlesungen über die Critik der reinen Vernunft, Fotomechan. Nachdr. Auflage. Bruxelles 1968, 14-15. „Ein einziges Ding, das durchgängig keine Verschiedenheit hat, wird nie einen allgemeinen Begriff ausmachen“ (a.a.O., 15). Demnach ist der Raum eine Anschauung. Wie man sehen kann, ist dieser Standpunkt nicht mit Anschaulichkeit im Sinne von Evidenz oder Vorstellbarkeit, sondern mit einer bloßen „logischen “ Frage verbunden: die allgemeinen Begriffe der traditionellen Logik sind nicht geeignet die Uniformität des Raumes auszudrücken.

57 Vgl. z.B. Krausser, Peter: The Operational Conception of ,reine Anschauung' (pure intuition) in Kant's Theory of Experience and Science. In: Studies In History and Philosophy of Science 3/1, 1972, 81-87: 82.

58 Vgl. z.B. Eine solche These wird auch von Michael Friedmann vertreten, obwohl durch eine andere auf Hermann von Helmholtz und Henri Poincaré gerichtete Strategie verwendet Friedman, Michael: Geometry, Construction, and Intuition in Kant and His Successors. In: Between Logic and Intuition: essays in Honor of Charles Parsons. Hrsg. von Gila Sher und Richard Tieszen. Cambridge 2002. Man behauptet natürlich, dass der Raum, von dem Kant spricht, der Raum der mathematischen Naturwissenschaft ist. 
Die Leibnizianer hätten nämlich behauptet, dass die Uniformität des Raumes, nach welcher Figuren auch nur solo numero (wegen der Lage im Raum) verschieden sein können, ${ }^{59}$ nur ein „verworrener Begriff“ 60 ist: Das würde aber nach Kant jede geometrische Erkenntnis a priori unmöglich machen: ${ }^{61}$ „Leibnitzens System über Raum und Zeit war, beyde in intellectuelle, aber verworrene Begriffe zu verwandeln. Aus diesen aber läßt sich nicht die Möglichkeit der Erkenntniß a priori begreifen, denn da müssen beide vorhergehen " (AA 23: Refl. XXX).

Das „transzendentale Argument“ Kants könnte also, selbstverständlich sehr pauschal, folgendermaßen zusammengefasst werden: Wenn die Teile des Raumes nicht alle gleichberechtigt wären, wenn der Raum also keine „reine Anschauung“ wäre, wäre die Geometrie (und ihre Anwendung auf die Mathematische Physik) ${ }^{62}$ unmöglich. Nur weil jeder Ort im Raum so gut wie jeder andere ist, kann man nämlich behaupten, dass eine synthetisch durchgeführte, geometrische Konstruktion $^{63}$ a priori an einem andern Ort ebenso gut als in diesem eintreten kann. Ge-

59 „Extensum dicitur uniforme, in quo non dantur nisi numero differentia: Difforme, in quo dantur quae intrinsece differunt, seu inter se dissimilia sunt.“ (Wolff: Philosophia prima sive Ontologia (1730), \$596).

60 „Extensionem et continuitatem in corpore nonnisi confuse percipimus“ (Wolff, Christian: Cosmologia generalis. In: Ders.: Gesammelte Werke. Hrsg. von Jean Ecole, 30 Bde., Hildesheim 1962, Bd. II.4, \224).

61 „Hinc in ipsa Mathesi acquiescimus in eo, quod confuse percipitur. Etenim in Geometria acquiescimus in notione extensionis, qualis a sensu in imaginationem derivatur" (Wolff, Christian: Psychologia rationalis. In: Ders.: Gesammelte Werke. Hrsg. von Jean Ecole, 30 Bde., Hildesheim 1962, Bd. II.6, \$161).

62 Die komplexe Beziehung zwischen Kants Raum- und Zeitlehre und das System der Grundsätze kann hier nicht erörtert werden. Es ist aber interessant zu bemerken, wie die Verbindung zwischen Symmetrien und Erhaltungssätze im Noehter-Theorem erstaunlicherweise Kants Behauptung zu „bestätigen“ scheint. Vgl. dazu Petitot, Jean: La philosophie transcendantale et le problème de l'objectivité. Paris 1991, 55.

63 Es ist ein Verdienst Jakko Hintikkas, gezeigt zu haben, dass sich Kant bei der Bestimmung der mathematischen Erkenntnis als „Konstruktion der Begriffe“ am geometrischen Beweisverfahren Euklids orientiert (vgl. Hintikka: On Kant's Notion of Intuition (Anschauung).; dazu vgl. auch Brittan, Gordon G., Jr.: Kant's Theory of Science. Princeton 1987, 51. Vgl. aber schon Beth, Evert Willem: Über Lockes ,allgemeines Dreieck““. In: KantStudien 48, 1956/57, 361-380). Wenn Kant behauptet, dass die Geometrie nicht „aus Begriffen“, sondern „aus der Construction der Begriffe“ (KrV, B 741) entsteht, scheint er einfach auf das Konstruktionsverfahren von Euklid zu verweisen: „The formation of generic concepts“, liest man in einer modernen Darstellung, „consists, then, in selecting from the plurality of objects only similar properties, while neglecting the rest [...] By contrast, Euclid's proofs focuses exactly on the features that the formation of generic concepts excludes. In Euclid's proofs, geometrical Figures are considered in their particularity [...] Therefore, a geometrical figure is not viewed from the perspective of its essential attributes, but from the perspective of the relations that it attains by being part of a certain spatial configuration “ (vgl. Harari, Orna: The Concept of Existence and the Role of Constructions in Euclid's Elements. In: Archive for History of Exact Sciences 57/1, 2003, 1-23: 14). Man kann also vermuten, dass Kant dieses Verfahren im Kopf hatte, als er behauptete, dass die Euklidische Geometrie nicht analytisch, sondern synthetisch ist. Das ist nämlich die Bedeutung der Konstruktionen bei Euklid: The „employment of constructions serves as a means of developing content; that is to say, it enables one to go beyond the content, which is given in the setting out stage, by placing the elementary geometrical figures 
rade deswegen kann „die einzelne hingezeichnete Figur“ nicht einfach als „Einzelfigur" dienen, sondern als ein „Schema “ gelten, die allen irgendwo anders, aber unter denselben „allgemeinen Bedingungen der Construction“ (KrV, B 742) bestimmten Figuren äquivalent ist. ${ }^{64}$ Nicht die auf dem Papier stehende Figur ist nämlich relevant, sondern die „Handlung der Construction“ (KrV, B 742), weil a priori behauptet werden kann, dass sie überall im Raum dieselben Resultate haben muss. ${ }^{65}$ Die Gleichheit und Ähnlichkeit aller Teile des Raumes ist also Ausdruck der Möglichkeit dafür, an verschiedenen Stellen des Raumes identische Konstruktionen vorzunehmen, da die Identität dieser Konstruktionen sich bei aller Verschiedenheit des Ausgangselementes feststellen lasse.

\subsection{Der Ähnlichkeitsbegriff bei Kant}

Kant scheint aber nicht nur zu behaupten, dass alle Teile des Raumes miteinander gleich und ähnlich sind, sondern auch dass sie zueinander ähnlich sind, d.h. dass es möglich ist, größere und kleinere Figuren zu konstruieren, ohne ihre geo-

(i.e., lines) in different spatial relations“ (a.a.O., 21). Sind gerade die Symmetrien des Euklidischen Raumes, die jene „infinity iterability of our process of construction“ ermöglichen (Friedman, Michael: Kant and the Exact Sciences. Cambridge, Mass. 1992, 61), der Notwendigkeit und Allgemeinheit der geometrischen Lehrsätze zugrunde liegt. Über die Verbindung zwischen geometrischen Konstruktionen und Raumsymmetrien vgl. auch Friedman: Geometry, Construction, and Intuition in Kant and His Successors, $210 \mathrm{ff}$.

64 Unter der unendlichen Literatur über das Thema, möchte ich immer noch auf Philonenko, Alexis: Lecture du schématisme transcendental. In: 200 Jahre Kritik der reinen Vernunft. Hrsg. von Joachim Kopper und Wolfgang Marx. Hildesheim 1981, 291-312 verweisen. Für jüngere Literatur vgl. Shabel, Lisa: Mathematics in Kant's Critical philosophy: Reflections on Mathematical Practice. New York 2003, 1. Teil; Shabel, Lisa: Kant's 'Argument from Geometry. In: Journal of the History of Philosophy 42/2, 2004, 195-215. Vgl. auch: Longuenesse, Béatrice: Kant and the Capacity to Judge Sensibility and Discursivity in the Transcendental Analytic of the Critique of Pure Reason. Princeton, NJ 1998, 287-291 und Friedman: Kant and the Exact Sciences, Kap. I.

65 Mit anderen Worten: „Greek geometers assumed that the (idealized) space in which their (idealized) figures existed was homogeneous for a given construction can be performed anywhere in it with the same results each lime." (Gray, Jeremy: Ideas of space. Oxford 1979, 29). Ähnliche Behauptungen sind in klassischen Texten zu finden: So z.B. Bernhard Bolzano: „Daher müssen mehrere ganz gleiche räumliche Dinge möglich seyn, von denen allen gleiche Prädicate gelten. Wenn also zu dem Puncte a irgend ein räumliches Ding A möglich ist, so muß auch zu dem verschiedenen Puncte b ein gleiches räumliches Ding B=A möglich seyn.“ (Bolzano, Bernhard: Betrachtungen über einige Gegenstände der Elementargeometrie. Prag 1804, 46). Noch deutlicher Hermann Graßmann: „Die Einfachheit des Raumes wird ausgesagt in dem Grundsatze: Der Raum ist an allen Orten und nach allen Richtungen gleich beschaffen, das heißt an allen Orten und nach allen Richtungen können gleiche Konstruktionen vollzogen werden." (Grassmann, Hermann: Die lineale Ausdehnungslehre ein neuer Zweig der Mathematik, dargestellt und durch Anwendungen auf die übrigen Zweige der Mathematik, wie auch auf die Statik, Mechanik, die Lehre vom Magnetismus und die Krystallonomie erläutert. Leipzig 1844, 35). 
metrischen Eigenschaften zu ändern. ${ }^{66}$ Obwohl Kant in seinen Hauptschriften das Problem der Ähnlichkeit nicht besonders ausführlich erörtert, ${ }^{67}$ ist es ihm selbstverständlich bekannt, dass in der Euklidischen Geometrie ähnliche Figuren, die sich nur in ihrer Seitenlänge unterscheiden, nicht verschiedene Figuren bilden, wenn man sie für sich betrachtet. Die einzelnen Gebilde werden in ihrer reinen „Qualität“ erfasst, ohne dass ein bestimmtes „Quantum“, ein absoluter Zahlenund Größenwert, für ihre Definition in Betracht käme („Ähnliche Dinge [sind] nur ihrer Größe nach unterschieden“, AA 16: 77; Refl. 1676). Diese Behauptung ist vielleicht wichtiger als man denken kann; wenn man eine Bestätigung finden will, dass für Kant die Euklidische Geometrie „a priori“ ist. Es muss nämlich wahrscheinlich, wenigstens implizit, hier gefunden werden, da nur in der Euklidischen Geometrie Figuren mit derselben Form, aber verschiedenen Größe, existieren. ${ }^{68}$ Was hier aber vor allem wichtig ist, ist, dass es auch für Kant bedeutet, da die Größe im Euklidischen Raum vollkommen relativ ist, die Unterscheidung von ähnlichen Figuren erst durch den Vergleich mit einem gegebenen Objekt möglich ist:

Daß alle Größe nur verhaltnismäßig sey u. es keine absolute Größe gebe ist daraus zu sehen. Ich messe am Himmel durch Erd diameter den Erd diameter durch Meilen die Meilen durch Füße diese durch das Verhältnis mit meinem Körper. (AA 20:47)

Kant scheint die Gegenüberstellung zwischen Begriff und Anschauung auch in Bezug auf die Relativität der Größe anzuwenden. Er behauptet, dass sich der Begriff „Fuß“ oder „Elle“ nicht definieren lässt; man kann nur eine bestimmte Strecke mit einem in der Anschauung gegebenen Objekt vergleichen, das wir als Standard festgelegt haben. Wir haben also, wie Kant ausdrücklich in der Kritik der Urtheilskraft betont, keinen „bestimmten Begriff von einer gegebenen Größe“ (KU, AA 05: 251; meine Hervorhebung), sondern es muss „die Schätzung der Größe des Grundmaßes bloß darin bestehen, daß man sie in einer Anschauung un-

66 Mit anderen Worten hat Kant damit die Apriorität der Symmetrien des Euklidischen Raumes vorausgesetzt, wie auch Jules Vuillemin bemerkt, vgl. Vuillemin, Jules: Physique et métaphysique kantiennes. Paris 1987, $51 \mathrm{f}$.

67 Vgl. dazu Sutherland, Daniel: Kant on Fundamental Geometrical Relations. In: Archiv für Geschichte der Philosophie 87, 2005, 117-158: 149.

68 Vgl. Der größte deutsche Mathematiker des 18. Jahrhunderts (und Kants Korrespondent), Johann Heinrich Lambert, erkannte, dass die Aufhebung des Parallelenpostulats zu der merkwürdigen Konsequenz führen muss, dass es analog zu den „absoluten“ Winkelmaßen (voller Winkel, gestreckter Winkel, rechter Winkel) auch „ein absolutes Maaß der Länge jeder Linien“ geben müsse (Lambert, Johann Heinrich: Theorie der Parallellinien (1766). In: Die Theorie der Parallellinien von Euklid bis auf Gauss: eine Urkundensammlung zur Vorgeschichte der nichteuklidischen Geometrie. Hrsg. von Friedrich Engel und Paul Stäckel. Leipzig 1895, 199. Das ist oft ein Argument für die Unplausibilität der nichteuklidischen Geometrie gewesen, da „bisher noch kein Mensch gezweifelt hat, daß es nehmlich kein solches absolutes Maaß gibt.“ (a.a.O., 200). Das Thema wurde im Bezug auf Kant im 19. Jahrhundert oft diskutiert: vgl. Renouvier, Charles: La philosophie de la règle et du compas. In: L'année philosophique (1891), 1-66: 43ff; Couturat, Louis: Note sur la Géométrie non euclidienne et la relativité de l'espace. In: Revue de Métaphysique et de Morale 1, 1893, 302-309. Vgl. auch Webb: Immanuel Kant and the Greater Glory of Geometry. $60 \mathrm{ff}$. 
mittelbar fassen [...] kann“ (KU, AA 05: 251; meine Hervorhebungen). ${ }^{69}$ Auch diese Behauptung Kants kann unsere Definition von Anschauungsbegriff bestätigen: Was eine „Elle“ ist, kann „mit Worten“ nicht erklärt werden, ebenso wie nicht „mit Worten“ definiert werden kann, was „links“ ist. ${ }^{70}$ Wesentlich ist also nochmals der Unterschied zwischen dem „Geben“ eines Gegenstandes durch individuelle Aufweisung einerseits, und dem „Definieren“ auf begrifflichen Wegen andererseits.

Die Größe bleibt trotzdem für Kant eine „innere“ Bestimmung einer Sache: „Daß etwas eine Größe (quantum) sei“, lässt sich nämlich „ohne alle Vergleichung mit andern“ erkennen; nur „wie groß es aber sei, erfordert jederzeit etwas anderes, welches auch Größe ist, zu seinem Maße“ und „die Größe dieser letztern immer wiederum etwas Anderes als Maß bedarf, womit sie verglichen werden könne“. ${ }^{71}$

69 Vgl. dazu Meerbote, Ralf: Kant on Intuitivity. In: Synthese 47/2, 1981, 203-228: 204. Für den Vergleich mit Leibniz vgl. De Risi: Geometry and Monadology: Leibniz's analysis situs and Philosophy of Space, 355 f., Fn. 44.

70 Das kann bestätigt werden, wenn man darauf achtet, dass der Ähnlichkeitsbegriff wohl eine wichtige Rolle in der Entstehungsgeschichte des Kantischen Beispiels der „inkongruenten Gegenstücke“ spielte. Interessant ist, dass in diesem Fall Christian Wolff ein guter Vermittler zwischen Leibniz und Kant zu sein scheint. In seinem Mathematischen Lexikon schreibt er bezugnehmend auf Leibniz' Ähnlichkeitsdefinition (vgl. dazu Poser, Hans: Die Bedeutung des Begriffs der Ähnlichkeit in der Metaphysik Christian Wolffs. In: Studia Leibnitiana 11, 1979, 62-81: „Denn die Grösse kann man einem Wohl geben und undeutlich in die Einbildung fassen, aber nicht mit Worten erklären und im Verstande deutlich begreifen “ (Wolff, Christian: Mathematisches Lexikon (1716). In: Ders.: Gesammelte Werke. Hrsg. von Jean Ecole, 30 Bde., Hildesheim 1962-, Bd. I.11, 179). Kant war sicher vertraut mit dieser Definition, die er in der vorkritischen Zeit kritisiert hatte (vgl. UD, AA 02: 277). Es scheint also nicht zufällig, dass in der Dissertatio Kant ähnliche Ausdrücke benutzt, etwa wenn er bemerkt, dass der Unterschied zwischen links und rechts „discursive describi s. ad notas intellectuales revocari nulla mentis acie possunt" [trotz aller Schärfe des Verstandes nicht begrifflich beschrieben, d.h. auf Verstandesmerkmale zurückgeführt werden] kann (MSI, AA 02: 403) oder dass es unmöglich ist einen solchen Unterschied „per omnia, quae notis menti per sermonem intelligibilibus efferre licet“ [durch Merkmale, die der Seele mittelst der Worte verständlich sind] zu fassen (MSI, AA 02: 403). Nach Lambert habe übrigens Wolff auch das Prinzip „quantitas dari, sed non per se intelligi potest" (Lambert: Theorie der Parallellinien (1766), 200) verwendet. Eine ähnliche Aussage findet nämlich in Wolffs Elementa Arithmeticae: „Cum Quantitate sine alio assumto per se non intelligi, sed tantum dari possit“ (Wolff, Christian: Elementa Matheseos Universae: Elementa Arithmeticae. In: Ders.: Gesammelte Werke. Hrsg. von Jean Ecole, 30 Bde., Hildesheim 1762-, Bd. II.29, \$26). Die Definition entspricht fast buchstäblich die schon erwähnte Aussage Kants, dass das, was links (oder rechts) ist „dari, sed non intelligi“ (MAN, AA 04: 484). Das bestätigt meines Erachtens, dass Kant die Frage gestellt hätte, ob „man im Raum, in dem wir leben, eine verbale Determinierregel angeben [kann], um festzulegen, was eine Rechts- und was eine Linksschraube sei“, oder ob es sich nicht vielmehr um eine „nichttheoretische“, „auf Zeigen beruhende Determination " handeln muss (Freudenthal, Hans: Mathematik in Wissenschaft und Alltag. München 1968, 243), gerade wie im Euklidischen Raum „die fundamentalen Maßeinheiten " nicht definiert (Freudenthal, Hans: Die Orientierung des Raumes. In: MathematischPhysikalische Semesterberichte 10, 1964, 161-174: 406), sondern nur gezeigt werden können.

71 Kant folgt aber Wolff und Baumgarten, auch wenn er behauptet, dass die „Quantität“ eine „innere Bestimmung“ sei, obwohl sie einen Vergleich erfordert. Kant unterscheidet 
Interessanterweise greift auch Kant in den Vorlesungen über die Metaphysik, die er im Wintersemester 1794/95 gehalten hat, auf das Gedankenexperiment der Ununterscheidbarkeit von ähnlichen Universen zurück, um eine solche Unmöglichkeit einer Definition des Maßes auszudrücken (V-Met-K3E/Arnoldt, AA 29.1,2: $835)$.

Nimmt man die Vorstellung an, einige Dinge im Universo würden immer kleiner, und alle übrigen dagegen unverändert, so stellt man sich ein Verhältniß vor, in welcher die kleinern gegen die größern bleiben [...]. Setzt man aber voraus, daß sich das ganze universum verändert habe, so ist keine Vergleichung weiter möglich, und also auch keine Veränderung der Größe denkbar [...] weil wir sie nicht mit einem dritten Gegenstande vergleichen können. (AA 29.1,2: 997)

Der Begriff der Größe ist demnach zwar absolut, „aber die magnitudo oder parvitas kann nie absolute erkannt werden, sondern nur durch relation “ (AA 29.1,2: 997). Kant benutzt das Argument, gerade um zu beweisen, dass man die Dinge an sich selbst nicht erkennen kann, sondern nur die Erscheinungen. In einer späten Reflexion (etwa Mai 1797) bemerkt Kant auf eine ähnliche Weise: „Daß sich alle ausgedehnte Wesen in der Welt in einen Wassertropfen oder ins unendliche noch kleineren Raum bringen lassen, beweiset die Idealität des Raums, wen alles immer als relativ, niemals absolut gros oder klein betrachtet wird" (AA 18: 669; Refl. 6344; vgl. auch AA 18: 705, Refl. 6398). Im Opus Postumum spricht Kant übrigens oft (vgl. AA 21: 196; 21: 338; 21: 501; 22: 4) davon, dass das ganze Universum in einer Nussschale Platz finden könne „ohne daß der mindeste Unterschied hierin anzutreffen" sei (AA 21: 197). Er führte diese Bemerkung auf Isaac Newton und auf den schweizerischen Chemiker und Meteorologen Jean-André Deluc zurück. Kant hatte aber auch die 1797 erschienene deutsche Übersetzung von Pierre-Simon Laplace Exposition du système $d u$ monde zur Kenntnis genommen, ${ }^{72}$ in der mit einem ähnlichen Gedankenexperiment die "Skaleninvarianz“ des Newtonschen Gravitationsgesetzes behauptet wird. ${ }^{73}$

\section{Konklusion: Symmetrie und Äquivalenz}

Um die Idealität des Raumes nachzuweisen, benutzt Kant also gerade dasselbe Leibniz'sche Gedankenexperiment, von dem man hier ausgegangen ist, ein Gedankenexperiment übrigens, auf das später unter anderen auch Hermann von Helm-

aber zwischen Quantitas und Quantum. In einer Anmerkung zum $\$ 69$ von Baumgartens Methaphysica, in der man zwischen qualitates und quantitates unterscheidet, bemerkt Kant: „besser quanta; daß etwas ein Qvantum sey, läßt sich absolut erkennen, wie groß aber (qvantitas), nur relativ" (vgl. auch AA 29: 991-992).

72 Laplace, Pierre Simon de: Darstellung des Weltsystems, übers. von Johann Karl Friedrich Hauff. Frankfurt am Mayn 1797.

73 „Une propriété remarquable de cette loi de la nature est que, si les dimensions de tous les corps de cet univers leurs distances mutuelles et leurs vitesses venoient à augmenter ou à diminuer proportionnellement, ils décriroient des courbes entièrement semblables à celles qu ils décrivent et leurs apparences seroient exactement les mêmes" (Laplace, Pierre Simon de: Exposition du système du monde, 2. Auflage. Paris 1799, 285). 
holtz, ${ }^{74}$ Henri Poincaré, ${ }^{75}$ Felix Hausdorff ${ }^{76}$ zugegriffen haben. Obwohl es hier um Textpassagen geht, die nicht den Hauptwerken Kants entnommen sind, können sie eine weitere Bestätigung unserer Forschungshypothese bieten, dass Leibniz' und Kants Auffassungen von Raum und Zeit von demselben Problem ausgehen, nämlich von dem Problem der Symmetrie: Eine Symmetrieoperation (Spiegelung, Drehung, Streckung usw.), um nochmals die Worte Hermann Weyls zu benutzen, „führt eine Figur in eine andere über, die, um mit Leibniz zu reden, ,von ihr ununterscheidbar ist, wenn jede der beiden Figuren für sich betrachtet wird" ". 77

Der Symmetriebegriff setzt damit eine Art der „Äquivalenz“-Setzung voraus, die zu den fundamentalsten Bestimmungen des modernen wissenschaftlichen Denkens überhaupt gehört. ${ }^{78}$ Hier wird die Gegenüberstellung, zwischen dem, was man als „dasselbe“ oder „nicht-dasselbe“ ansieht, von einem neuem Standpunkt betrachtet: die geometrische „Gleichberechtigkeit“ von Figuren wird von der logischen „Identität“ von Begriffen unterschieden und getrennt. Kant scheint natürlich dem Raum a priori die Symmetrie des Euklidischen Raums (in dem die Lage und die Größe einer Figur relativ sind) zuzuschreiben, was aber - wie es sich später gezeigt hat - nicht selbstverständlich ist (in der sphärischen und pseudosphärischen Geometrie z.B. ist zwar die Lage relativ, aber nicht die Größe). Relevant ist aber vielmehr, dass der Raum bestimmte Symmetriebedingungen überhaupt erfüllen muss, wenn die wissenschaftliche Erkenntnis der Natur möglich sein soll. ${ }^{79}$ Man kann nämlich behaupten, dass ohne solche „Äquivalenz“, die der Symmetriebegriff voraussetzt, die Naturwissenschaft unmöglich wäre. ${ }^{80}$ Wie eine geome-

74 Vgl. Helmholtz, Hermann: Über den Ursprung und die Bedeutung der geometrischen Axiome. In: Vorträge und Reden. Braunschweig/Wiesbaden 1903, Bd. 1, 23-51:44.

75 Vgl. Poincaré, Henri: Wissenschaft und Methode. Leipzig 1914, $81 \mathrm{f}$.

76 Vgl. Mongré: Das Chaos in kosmischer Auslese. Ein erkenntnisskritischer Versuch, 38.

77 Weyl: Symmetrie, 26.

78 Vgl. Rosen: Symmetry Rules: How Science and Nature Are Founded on Symmetry, 96.

79 Es sei hier also vorübergehend bemerkt, dass, wenn man nach einer „Widerlegung“ der Kantischen Raumlehre sucht, nicht in der bloßen Entdeckung der nichteuklidischen Geometrien zu finden ist, sondern in der von Bernhard Riemann in seinen 1854 gehaltenen Habilitiationsvortrag (Riemann, Bernhard: Über die Hypothesen, welche der Geometrie zugrunde liegen. In: Ders.: Gesammelte mathematische Werke, wissenschaftlicher Nachlass und Nachträge. Hrsg. von Raghavan Narasimhan und Heinrich Weber, Berlin 1990, 304-319) vorgeschlagenen Raumauffassung. In Riemannschen Räumen ist die Raumstruktur im allgemeinsten Fall in jedem Punkte und nach verschiedenen Richtungen unterschiedlich und besitzt deshalb keine Symmetrie, wenn nicht die bloße Identität. Vgl. dazu Giovanelli, Marco: Kant, Helmholtz, Riemann und der Ursprung der geometrischen Axiome. In: Philosophia naturalis 45/2, 2008, 236-269.

80 Wir beschränken uns auf die sogenannten „globalen“ Symmetrien; für den Unterschied von globaler und lokaler Symmetrie vgl. z. B. 't Hooft, Gerardus: Gauge Theories of the Forces Between Elementary Particles. In: Scientific American 242/6, 1980, 104-138. In der Raum-Zeit der allgemeinen Relativitätstheorie, wie in der Riemannschen Geometrie, gibt es z.B. keine nicht-Triviale globale Symmetrie. Die allgemeine Relativitätstheorie wird also manchmal als die erste Theorie mit einer lokalen Symmetrie interpretiert. (Vgl. dazu Auyang, Sunny Y.: How is Quantum Field Theory Possible? Oxford/New York 1995, 38) Die Entwicklung der Physik des 20. Jahrhunderts, insbesondere die moderne 
trische Figur sich nicht verändert, wenn man sie von einem Punkt zum anderen bewegt, so müssen Nachbauten von physikalischen Apparaten zu einem anderen Raumpunkt genauso wie die Urbilder laufen. Sie sind also für sich betrachtet nicht zu unterscheiden, weil uns keine Beobachtung, Messung usw. besagen kann, ob es sich um die ursprüngliche Apparatur handelt oder um die verschobene. In Gegensatz zur Geometrie, funktionieren aber in der Physik eine Apparatur und ihr Modell im verkleinerten Maßstab nicht notwendigerweise gleich, da man aufgedeckt hat, dass es eine absolute Normallänge in der Konstitution des Atoms gibt (zum Nachbau einer doppelt so großen Apparatur bräuchte man doppelt so große Atome, was unmöglich ist). In der älteren Physik musste man solch eine Eichung zeigen. In der neueren kann man sie verbal mitteilen. Noch erstaunlicher war das Resultat des 1956 gehaltenen Wu-Experiments (nach Chien-Shiung Wu), das die bis dahin vorherrschende Überzeugung erschütterte, dass die Naturgesetze links und rechts nicht unterscheiden können (eine Apparatur und ihr Spiegelbild müssten genau gleich funktionieren). ${ }^{81}$ Vor diesem Experiment wäre es nötig gewesen zu zeigen, welche die Rechtsschraube ist, heute könnte man sich dagegen verbal verständigen.

Welche Symmetrien in der Natur gelten, muss also sicher die Erfahrung entscheiden und man braucht sich nicht länger, wie Kant es tat, darüber zu wundern, dass die Gruppe der geometrischen Symmetrien umfassender ist als die der physikalischen. ${ }^{82}$ Dass es Symmetrien in der Natur überhaupt gibt, macht aber die wissenschaftliche Erfahrung erst möglich. Wäre die Natur vollkommen asymmetrisch, hingen z. B. die Naturgesetze vom Ort ihrer Anwendung ab, wären Experimente nicht wiederholbar, und die wissenschaftliche Naturerkenntnis wäre unmöglich. ${ }^{83}$ Aus diesem Standpunkt könnte die oft zitierte Behauptung von Hermann Weyl verstanden werden, dass „alle a-priori-Behauptungen in der Physik ihren Ursprung in der Symmetrie " 84 haben.

Quantenfeldtheorie, bestätigt also dass die Symmetrien als, wie man mit den Worten Steven Weinbergs sagen könnte, „a priori principles of universal validity“ (Weinberg: Conceptual Foundations of the Unified Theory of Weak and Electromagnetic Interactions, 215) angesehen werden können.

81 Vgl. dazu z.B. Freudenthal: Mathematik in Wissenschaft und Alltag, 243ff; Feynman, Richard Phillips: Physikalische Fingerübungen für Fortgeschrittene. München 2004, 84 ff.; in Bezug auf Kant vgl. z.B. Gartner, Martin: The Ozma Problem and the Fall of Parity. In: The Philosophy of Right and Left. Incongruent Counterparts and the Nature of Space. Hrsg. von James Van Cleve und Robert E. Frederick. Dordrecht 1991.

82 Vgl. Weyl: Symmetrie, 130.

83 Vgl. z. B. Wigner: Symmetries and Reflections: Scientific Essays of Eugene P. Wigner, 4, $19,29,225 \mathrm{f}$.

84 Weyl: Symmetrie, 126. 